Commun. Math. Phys. 108, 535-589 (1987)

Communications in

Mathematical

Physics

(C) Springer-Verlag 1987

\title{
Hyperkähler Metrics and Supersymmetry
}

\author{
N. J. Hitchin ${ }^{1}$, A. Karlhede ${ }^{2, \star}$, U. Lindström ${ }^{2}$, and M. Roček ${ }^{3, \star \star}$ \\ ${ }^{1}$ Mathematical Institute, University of Oxford, 24-29 St. Giles, Oxford OX1 3LB, \\ United Kingdom \\ ${ }^{2}$ Institute of Theoretical Physics, University of Stockholm, Vanadisvägen 9, \\ S-11346 Stockholm, Sweden \\ ${ }^{3}$ Institute for Theoretical Physics, State University of New York, Stony Brook, NY 11794, USA
}

\begin{abstract}
We describe two constructions of hyperkähler manifolds, one based on a Legendre transform, and one on a symplectic quotient. These constructions arose in the context of supersymmetric nonlinear $\sigma$-models, but can be described entirely geometrically. In this general setting, we attempt to clarify the relation between supersymmetry and aspects of modern differential geometry, along the way reviewing many basic and well known ideas in the hope of making them accessible to a new audience.
\end{abstract}

\section{Table of Contents}

1. Introduction . . . . . . . . . . . . . . . . . . . . . . . . . . . . . . . 536

2. Construction of New Hyperkähler Metrics . . . . . . . . . . . . . . . . . 538

(A) Legendre Transform . . . . . . . . . . . . . . . . . . . . . . . . . . . 538

(B) Symplectic Quotient . . . . . . . . . . . . . . . . . . . . . . . . . . . 540

(C) Quotients and Legendre Transforms . . . . . . . . . . . . . . . . . . . 542

3. Geometric Interpretation . . . . . . . . . . . . . . . . . . . . 543

(A) Quotient Manifolds . . . . . . . . . . . . . . . . . . . . . . . . 543

(B) Symplectic Quotients . . . . . . . . . . . . . . . . . . . . . . . . . . 544

(C) Kähler Quotients . . . . . . . . . . . . . . . . . . . . . . . . . . . . 547

(D) Hyperkähler Quotients. . . . . . . . . . . . . . . . . . . . . . . . . . 548

(E) Kähler Potentials . . . . . . . . . . . . . . . . . . . . . . . . . . . . 550

(F) Twistor Spaces . . . . . . . . . . . . . . . . . . . . . . . . . . . . 554

(G) The Legendre Transform . . . . . . . . . . . . . . . . . . . . . . . 561

4. Nonlinear $\sigma$-Models . . . . . . . . . . . . . . . . . . . . . . . . . . . . . . 565

(A) Basics . . . . . . . . . . . . . . . . . . . . . . . . . . . . . . . 565

(B) Duality . . . . . . . . . . . . . . . . . . . . . . . . . . . 567

(C) Gauging . . . . . . . . . . . . . . . . . . . . . . . . . . . . . . 568

(D) $C P(1) \ldots \ldots \ldots 69$

* Supported by the Swedish Natural Science Research Council

$\star \star$ Research supported by the National Science Foundation under Contract No. PHY 8109110 A-01 and PHY 85-07627 
5. Supersymmetry . . . . . . . . . . . . . . . . . . . . 570

(A) Introduction . . . . . . . . . . . . . . . . . . . . . 570

(B) Preliminaries . . . . . . . . . . . . . . . . . . . . . . . . . . . . . . 572

(C) Superalgebras and Superspace . . . . . . . . . . . . . . . . . . . . 572

(D) Superfields and Spinor Derivatives. . . . . . . . . . . . . . . . . . . . . . . 573

(E) $N=1$ Scalar Superfields . . . . . . . . . . . . . . . . . . . . . . . . . 574

(F) $N=1$ Gauge Superfields . . . . . . . . . . . . . . . . . . . . . . . . 575

(G) $N=1$ Supersymmetry Transformations . . . . . . . . . . . . . . . . . . . 576

(H) $N=2$ Spinor Derivatives and Superfields . . . . . . . . . . . . . . . . . . 576

(I) $\quad N=2$ Components and Supersymmetry Transformations . . . . . . . . . . 577

(J) $N=2$ Gauge Fields . . . . . . . . . . . . . . . . . . . . . . . . . . . 578

(K) $N=2$ Gauge Transformations. . . . . . . . . . . . . . . . . . . . . . . 579

(L) $N=1$ form of $N=2$ Superfields . . . . . . . . . . . . . . . . . . . 579

(M) $N=4$ Supersymmetry . . . . . . . . . . . . . . . . . . . . . . . . . . . . . . . 580

(N) Actions in Superspace . . . . . . . . . . . . . . . . . . . . . . . . . . 581

6. The Supersymmetric Construction of Hyperkähler Metrics . . . . . . . . . . . . 582

(A) The Supersymmetric Legendre Transform Construction. . . . . . . . . . . . 582

(B) Gauging of Isometries and the Quotient Construction . . . . . . . . . . . . 585

References . . . . . . . . . . . . . . . . . . . . . . 588

\section{Introduction}

In this article, we describe two constructions of new hyperkähler manifolds ${ }^{1}$. These constructions, which arose first in the context of certain supersymmetric models $[4,5]$, have a clear geometric meaning. We attempt to clarify the relation of supersymmetry and modern differential geometry (at least in this particular context), and to break down the language barrier between geometers and supersymmetrists via the description of these constructions. To this end, we review many basic and well known notions in terms intended to make them accessible to a new audience.

After sketching the structure of the article we end this section by reviewing basic notions of Kähler and hyperkähler geometry and establishing some notation. In the following section, we describe our constructions, without using supersymmetry, and give examples. The first construction uses a Legendre transform to relate the Kähler potentials of certain hyperkähler manifolds to a linear space. The second construction is based on a symplectic quotient of a hyperkähler manifold. We give a number of examples. We also discuss in detail the cases when both constructions are applicable. In Sect. 3, we give some background needed to explain the geometric meaning of the constructions: quotients, symplectic and Kähler quotients, and twistor theory, and then give the geometric interpretation. In Sect. 4 we describe nonlinear $\sigma$-models and related material needed as a background for subsequent sections. In Sect. 5, we describe essential aspects of supersymmetry and, in Sect. 6, we use supersymmetry to derive the constructions. The most common use of various index types is indicated in Table 1.

On a $2 n$ (real) dimensional Kähler manifold (see discussion above (3.20)) we choose holomorphic coordinates $z^{q}, \bar{z}^{\bar{q}}, q=1, \ldots, n$ in which the complex structure is

$$
\mathbf{I}=i\left(\begin{array}{cc}
\delta_{q}^{p} & 0 \\
0 & -\delta_{\bar{q}}^{\bar{p}}
\end{array}\right) .
$$

${ }^{1}$ Reviews of Kähler and hyperkähler geometry for physicists can be found in [1-3] 
Table 1. Most common use of indices throughout the paper. All dimensions indicated are real

\begin{tabular}{lll}
\hline Indices & Description & Range \\
\hline$i, j, \ldots$ & Coordinates on a manifold $M$ & $1, \ldots, \operatorname{dim} M$ \\
$p, q, \ldots$ & Holomorphic coordinates on a complex manifold $M$ & $1, \ldots, \frac{1}{2} \operatorname{dim} M$ \\
$\bar{p}, \bar{q}, \ldots$ & Antiholomorphic coordinates & \\
$i, j, \ldots$ & Generic; & Varied \\
& $\begin{array}{l}\text { Often, a restricted range of coordinates } \\
\text { on a hyperkähler manifold } M\end{array}$ & $1, \ldots, \frac{1}{4} \operatorname{dim} M$ \\
$a, b, \ldots$ & Spacetime coordinates & Usually $1,2,3$ \\
$\alpha, \beta, \ldots$ & Spacetime spinor indices & Usually 1,2 \\
$A, B, \ldots$ & Basis elements of a Lie algebra $\mathfrak{g}$ & $1, \ldots, \operatorname{dimg}$ \\
\hline
\end{tabular}

In these coordinates the metric $g=g_{i j} d x^{i} \otimes d x^{j}\left(\left\{x^{i}\right\}=\left\{z^{p}, \bar{z}^{\bar{q}}\right\}\right)$ is expressed in terms of a Kähler potential $K$,

$$
g=2 K_{q \bar{p}} d z^{q} \otimes d \bar{z}^{\bar{p}},
$$

where $K_{q \bar{p}} \equiv \partial^{2} K / \partial z^{q} \partial \bar{z}^{\bar{p}}$ and the Kähler form is the nondegenerate symplectic 2-form

$$
\omega=2 i \partial \bar{\partial} K=2 i K_{q \bar{p}} d z^{q} \wedge d \bar{z}^{\bar{p}}=-g_{i j} \mathbf{I}_{k}^{j} d x^{i} \wedge d x^{k} .
$$

A vector field is Hamiltonian, holomorphic or Killing if it preserves $\omega, \mathbf{I}$ or $g$ respectively

$$
L_{X} \omega=0, \quad L_{X} \mathbf{I}=0, \quad \text { or } \quad L_{X} g=0,
$$

where $L_{X}$ is the Lie derivative along $X$. Any two of these conditions imply the third. In particular, the last condition implies that a Killing vector fulfills Killing's equation

$$
X_{i ; j}+X_{j ; i}=0 \text {. }
$$

We can expand an arbitrary Killing vector field over a basis of Killing vector fields $k_{A}$ that generate the Lie algebra of the isometry group

$$
\begin{gathered}
X=X^{A} k_{A}, \\
{\left[k_{A}, k_{B}\right]=c_{A B}{ }^{C} k_{C},}
\end{gathered}
$$

where $X^{A}$ and $c_{A B}{ }^{C}$ are constants. A holomorphic Killing vector in holomorphic coordinates can be split into a holomorphic and an antiholomorphic piece

$$
\frac{1}{2}(X-i \mathbf{I} X)=X^{A} k_{A}^{p}(z) \partial_{p}, \quad \frac{1}{2}(X+i \mathbf{I} X)=X^{A} \bar{k}_{A}^{\bar{p}}(\bar{z}) \bar{\partial}_{\bar{p}} .
$$

On any manifold with a symplectic structure $\omega$ the relation $L_{X} \omega=0$ is the integrability condition for the existence of a Hamiltonian function $\mu^{X}$ for the vector field $X$,

$$
X^{i} \omega_{i j}=\mu^{X}, j .
$$

When the manifold is Kähler, a holomorphic Hamiltonian vector field is a Killing vector field and (1.9) becomes (in holomorphic coordinates)

$$
\omega_{\bar{q} p} X^{A} \bar{k}_{A}^{\bar{q}}=-i K_{p \bar{q}} X^{A} \bar{k}_{A}^{\bar{q}}=\mu^{X}, p, \quad \mu^{X} \equiv X^{A} \mu_{A} .
$$

In this case we call $\mu^{X}$ a Killing potential and $\left\{\mu_{A}\right\}$ is a basis corresponding to $\left\{k_{A}\right\}$. Equation (1.10) defines the Killing potential only up to a real constant. Up to 
abelian factors in the algebra, we fix this constant by requiring that $\mu^{X}$ is equivariant,

$$
X \mu^{Y}=\mu^{[X, Y]} \Leftrightarrow k_{A}^{p} \mu_{B, p}+\bar{k}_{A}^{\bar{p}} \mu_{B, \bar{p}}=c_{A B}{ }^{C} \mu_{C} .
$$

If the relevant Lie algebra cohomology class is nontrivial, there may be obstructions, i.e., irremovable constants on the right-hand side of (1.11), (for examples, see $[6,3])$. If the Lie algebra is semisimple, we can compute $\mu_{A}$ algebraically from (1.11) using (1.10). The potentials $\mu_{A}$ are maps from the manifold to the dual of the Lie algebra; when $\mu_{A}$ are equivariant we call them moment maps. [See discussion from (3.8) to (3.13).]

A $4 n$ (real) dimensional hyperkähler manifold has three independent complex structures $\mathbf{I}, \mathbf{J}$, and $\mathbf{K}$ satisfying the quaternion algebra identities:

$$
\mathbf{I}^{2}=\mathbf{J}^{2}=\mathbf{K}^{2}=-1, \quad \mathbf{I J}=-\mathbf{J} \mathbf{I}=\mathbf{K}, \quad \text { etc. }
$$

We call the corresponding symplectic forms $\omega_{P}, P=1,2,3, \omega_{P}=-(g \mathbf{I}, g \mathbf{J}, g \mathbf{K})$. If we choose holomorphic coordinates with respect to $\mathbf{I}$, the combinations

$$
\omega^{ \pm}=\left(\omega_{2} \pm i \omega_{3}\right), \quad \omega^{+}=\omega_{p q}^{+}(z) d z^{p} \wedge d z^{q}, \quad \omega^{-}=\overline{\omega^{+}}
$$

are holomorphic and antiholomorphic. A triholomorphic Killing vector $X$ preserves all three symplectic forms

$$
L_{X} \omega_{P}=0 .
$$

This implies the existence of three independent Killing potentials $\mu_{(P)}^{X}=X^{A} \mu_{A}^{(P)}$ for a Killing vector field $X$. We can choose one to be defined with respect to the Kähler form, cf. (1.10), and holomorphic and antiholomorphic potentials defined with respect to $\omega^{ \pm}$:

$$
\omega_{p q}^{+}(z) k_{A}^{p}(z)=\mu_{A, q}^{+}(z), \quad \mu^{-}=\overline{\mu^{+}} .
$$

Again, Eq. (1.15) defines $\mu^{ \pm}$only up to a complex constant, which we fix (except for abelian factors) by requiring equivariance, $\mathrm{cf}$ (1.11):

$$
X \mu_{(P)}^{Y}=\mu_{(P)}^{[X, Y]} \Rightarrow k_{A}^{p} \mu_{B, p}^{+}=\frac{1}{2} c_{A B}{ }^{C} \mu_{C}^{+} \text {, etc. }
$$

As before, there may be obstructions to (1.16).

\section{Constructions of New Hyperkähler Metrics}

\section{(A) Legendre Transform}

We start with $3 n$ real dimensional flat space $E=R^{n} \times C^{n}$ (we may choose coordinates $\left.x^{i} \in R, z^{i} \in C, i=1, \ldots, n\right)$. We consider a real function $F: E \rightarrow R$, i.e., $F\left(x^{i}, z^{i}, \bar{z}^{i}\right)$, that satisfies the system of linear differential equations

$$
F_{x^{i} x^{j}}+F_{z^{i} \bar{z} j}=0 .
$$

This implies that $F$ satisfies the three-dimensional Laplace equation on all threedimensional subspaces $(X, Z, \bar{Z})$ with $X=a_{i} x^{i}, Z=a_{i} z^{i}, \bar{Z}=a_{i} \bar{z}^{i}$ determined by an $n$-vector with real components $a_{i}$. A characterization of $F$, equivalent to (2.1), is as a contour integral in an auxiliary variable $\zeta[7]$,

$$
F\left(x^{i}, z^{i}, \bar{z}^{i}\right)=\operatorname{Re} \frac{1}{2 \pi i} \oint_{c} d \zeta G\left(\eta^{i}(\zeta), \zeta\right),
$$


where

$$
\eta^{i}=z^{i}-\zeta x^{i}-\zeta^{2} \bar{z}^{i}
$$

Then the n-dimensional Legendre transform of $F$ with respect to the real coordinates $x^{i}$ gives the Kähler potential $K\left(u^{i}, \bar{u}^{i}, z^{i}, \bar{z}^{i}\right)$ of a $4 n$ real dimensional hyperkähler manifold [5]:

$$
K\left(u^{i}, \bar{u}^{i}, z^{i}, \bar{z}^{i}\right)=F\left(x^{i}, z^{i}, \bar{z}^{i}\right)-\left(u^{i}+\bar{u}^{i}\right) x^{i},
$$

where $x^{i}$ is a function of $z^{i}, \bar{z}^{i}$, and $u^{i}+\bar{u}^{i}$ determined by

$$
\frac{\partial F}{\partial x^{i}}=u^{i}+\bar{u}^{i}
$$

The metric of the manifold is computed as usual from the Kähler potential (1.2)

$$
\frac{1}{2} g=K_{u^{i} \bar{u}^{j}} d u^{i} \otimes d \bar{u}^{j}+K_{u^{i} \bar{z}^{j}} d u^{i} \otimes d \bar{z}^{j}+K_{z^{i} \bar{u}^{j}} d z^{i} \otimes d \bar{u}^{j}+K_{z^{i} \bar{z}^{j}} d z^{i} \otimes d \bar{z}^{j} .
$$

From $(2.4,5)$, the Kähler potential is independent of $u^{i}-\bar{u}^{i}$, and hence the metric (2.6) has $n$ abelian holomorphic Killing vectors [cf. (1.4)-(1.8)]. (Actually, the Killing vectors are triholomorphic, see below [22].)

The line element can be computed explicitly in nonholomorphic coordinates even when Eq. (2.5) cannot be solved explicitly for $x^{i}$. We use the original coordinates $x^{i}, z^{i}$, and $\bar{z}^{i}$, and $n$ additional real coordinates, e.g., $y^{i}=i\left(\bar{u}^{i}-u^{i}\right)$. The line element in these coordinates is (2.6) with [8]:

$$
\begin{aligned}
K_{u^{i} \bar{u}^{j}} & =-\left(F_{x^{j} x^{i}}\right)^{-1}, K_{u^{i} \bar{z}^{j}}=\left(F_{x^{k} x^{i}}\right)^{-1} F_{x^{k} \bar{z}^{j}}, \\
K_{z^{i} \bar{z}^{j}} & =-\left(F_{x^{i} x^{j}}+F_{z^{i} x^{k}}\left(F_{x^{l} x^{k}}\right)^{-1} F_{x^{l} \bar{z}^{j}}\right), \\
2 d u^{i} & =F_{x^{i} x^{j}} d x^{j}+F_{x^{i} z^{j}} d z^{j}+F_{x^{i} \bar{z}^{j}} d \bar{z}^{j}+i d y^{i} .
\end{aligned}
$$

The construction also explicitly generates the quaternionic structure of the hyperkähler manifold [cf. (1.12)]. The corresponding 2-forms are [cf. $(1.3,13)]$

$$
\begin{aligned}
\frac{1}{2} \omega^{1} & =i\left(K_{u^{i} j} d u^{i} \wedge d \bar{u}^{j}+K_{u_{\bar{z}}^{i} j} d u^{i} \wedge d \bar{z}^{j}+K_{z^{i} \bar{u}^{j}} d z^{i} \wedge d \bar{u}^{j}+K_{z^{i} \bar{z}^{j}} d z^{i} \wedge d \bar{z}^{j}\right), \\
\frac{1}{4} \omega^{+} & =d u^{i} \wedge d z^{i}-\left(K_{u^{i} \bar{u}^{j}}\right)^{-1} K_{\bar{u}^{j} z^{k}} d z^{i} \wedge d z^{k}, \\
\omega^{-} & =\overline{\omega^{+}} .
\end{aligned}
$$

The isometries generated by

$$
k_{(i)}=i\left(\frac{\partial}{\partial u^{i}}-\frac{\partial}{\partial \bar{u}^{i}}\right)
$$

clearly preserve the 2-forms $\omega^{ \pm}$, and hence are triholomorphic [cf. (1.14)].

We now describe several examples. The basic ingredients are the different descriptions of flat four dimensional space given by the functions $G(\eta, \zeta)$ and contours $C$ [see (2.2)] [7]:

$$
G_{1}=-\frac{1}{2 \zeta^{3}} \eta^{2} \quad \begin{aligned}
& \text { with any contour enclosing the origin once } \\
& \text { in anti-clockwise direction }
\end{aligned}
$$

and

$$
G_{2}=\frac{1}{\zeta^{2}} \eta \ln \eta \quad \text { with the contour in Fig. } 1 .
$$

The corresponding function $F(x, z, \bar{z}) \equiv F(\vec{r}), r^{2}=x^{2}+4 z \bar{z}$, are:

$$
\begin{gathered}
F_{1}=-\frac{1}{2} x^{2}+z \bar{z} \\
F_{2}=r-x \ln (x+r)+\frac{1}{2} x \ln (4 z \bar{z}) .
\end{gathered}
$$




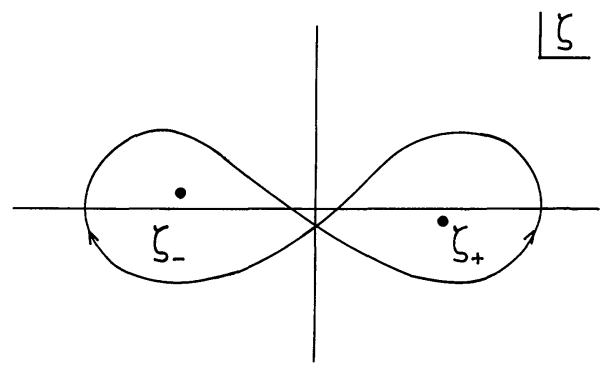

Fig. 1. Contour of integration. $\zeta_{ \pm}$are the roots of $\eta(\zeta): \eta=-z\left(\zeta_{-} \zeta_{+}\right)\left(\zeta_{-} \zeta_{-}\right)$, $\zeta_{ \pm}=-(1 / 2 z)(x \pm r), r^{2} \equiv x^{2}+4 z \bar{z}$

The last term in $(2.13), \frac{1}{2} x \ln (4 z \bar{z})=\frac{1}{2} x(\ln (2 z)+\ln (2 \bar{z}))$, can be dropped, as, after the Legendre transform $(2.4,5)$, it merely generates a holomorphic coordinate transformation, $u \rightarrow u+\frac{1}{2} \ln (2 z)$.

A number of interesting manifolds can be constructed as various superpositions of $F_{1}$ and $F_{2}$. For example, the multi-Eguchi-Hanson family of self-dual instantons $[9,10]$ is found by superposing $F_{2}$ with itself about different (mass) points $\vec{\varrho}_{A}$ :

$$
F_{\mathrm{EH}}=\sum_{A=1}^{m+1} F_{2}\left(\vec{r}-\vec{\varrho}_{A}\right) \text {. }
$$

The Taub-NUT family of self-dual instantons [10] is found by adding an $F_{1}$ as well:

$$
F_{\mathrm{TN}}=F_{1}(\vec{r})+\sum_{A=1}^{m} F_{2}\left(\vec{r}-\vec{\varrho}_{A}\right) .
$$

In higher dimensions, the metrics due to Calabi [11] are constructed as follows:

$$
F_{C}=\sum_{i=1}^{m} F_{2}\left(\vec{r}^{i}\right)+F_{2}\left(\vec{\varrho}-\sum_{i=1}^{m} \vec{r}^{i}\right) .
$$

New metrics can be found by adding more mass points to (2.16) as in (2.14), and by adding $F_{1}$ terms as in $(2.15)[5,8]$.

The global properties of manifolds constructed by the Legendre transform have not been investigated in general. However, the examples given here can also be constructed by the quotient construction discussed below, which allows a global analysis.

\section{(B) Symplectic Quotient}

In this construction, we start with a hyperkähler manifold $M$, for example, $M=C^{n} \times C^{n}$, with one or more triholomorphic isometries acting on it. A new, lower dimensional, hyperkähler manifold is constructed as the quotient of a real subspace of $M$ by some subgroup of the isometry group. Alternatively we can view the construction as the quotient of a holomorphic subspace of $M$ by a subgroup of the complexification of the isometry group. (The geometric pictures of these alternatives are presented in Sect. 3.) In either case the subspace is defined by a set of algebraic equations. If the equations are well behaved (free of cusps, etc.) and if the quotient group is compact and acts freely (i.e., has trivial isotropy 
group) on the subspace the equation define, then the manifold is complete. Of course, the construction can be used even when the manifold is incomplete, e.g., has singularities; in particular, if the isotropy group on some subspace has only a finite number of elements, the resulting quotient space is a hyperkähler orbifold. We work with holomorphic coordinates $z^{p}, \bar{z}^{p}, p=1, \ldots, 2 n$, cf. (1.1-3).

We assume that the manifold has triholomorphic Killing vectors $X=X^{A} k_{A}$ [cf. (1.6-8)] where $k_{A}=k_{A}^{p} \partial_{p}+\bar{k}_{A}^{\bar{p}} \bar{\partial}_{\bar{p}}, A=1, \ldots, k$ is a basis for the Lie algebra of the subgroup of the isometry group by which we wish to divide.

We can consider the construction as the quotient of the real subspace $\mu_{A}^{(P)}=0$ [cf. (1.14-15)] by the quotient group. This quotient is well defined because moment maps are equivariant (1.16). The holomorphic view of the construction gives the Kähler potential explicitly. The holomorphic subspace is defined by the conditions

$$
\mu_{A}^{+}=\mu_{A}^{-}=0 .
$$

Note that because $\left(\mu^{-}\right) \mu^{+}$is (anti-) holomorphic the constraint (2.17) is preserved by the complexification of the isometry group, which acts by

$$
z^{\prime}=e^{\Lambda} z, \quad \bar{z}^{\prime}=e^{\bar{\Lambda}} \bar{z},
$$

where

$$
\Lambda=\Lambda^{A}(z) k_{A}^{p} \partial_{p}, \quad \bar{\Lambda}=\bar{\Lambda}^{A}(\bar{z}) \bar{k}_{A}^{\bar{p}} \bar{\partial}_{\bar{p}} .
$$

The relation $\mu_{A}^{(1)}=0$ is preserved only by the real action. We introduce a real vector field $V$ that is an (in general different) Killing vector at each point:

$$
V=V^{A}(z, \bar{z})\left(k_{A}^{p} \partial_{p}+\bar{k}_{A}^{\bar{p}} \bar{\partial}_{\bar{p}}\right) \text {. }
$$

We also introduce the complementary real vector field $Y$ that together with $V$ generates the complexified group at each point:

$$
Y=-\frac{1}{2} \mathbf{I} V=-\frac{i}{2} V^{A}\left(k_{A}^{p} \partial_{p}-\bar{k}_{A}^{\bar{p}} \bar{\partial}_{\bar{p}}\right) .
$$

We use $V^{A}$ to enlarge the submanifold $\mu_{A}^{(1)}=0$ to a higher dimensional submanifold

$$
e^{Y} \mu_{A}^{(1)}=0,
$$

which is preserved by the complexified action provided that $V$ is chosen appropriately. The $k$ equations (2.22) are to be solved for the $k$ components $V^{A}$, which determines the vector field $V$. (In $e^{Y}, Y$ does not act on $V^{A}$.) The Kähler potential $\hat{K}$ of the new manifold is defined on the quotient of the subspace (2.17) in terms of auxiliary real functions $V^{A}(z, \bar{z})[3]$ :

where $\mu^{V}=V^{A} \mu_{A}^{(1)}$.

$$
\hat{K}=K(z, \bar{z})-\int_{0}^{1} d t e^{t Y} \mu^{V}=K(z, \bar{z})-\frac{e^{Y}-1}{Y} \mu^{V},
$$

Equation (2.22) can be found by treating $V$ as an independent variable and extremizing $\hat{K}$ with respect to arbitrary variations of it:

$$
\frac{\delta \hat{K}}{\delta V^{A}}=0 \Leftrightarrow e^{Y} \mu_{A}^{(1)}=0 .
$$

This gives the symplectic quotient on hyperkähler manifolds. 
As an example we consider $M=$ flat $C^{n} \times C^{n}$ with coordinates $\left(\zeta^{i}, \xi^{i}\right) i=1, \ldots, n$ [5]. The holomorphic symplectic structure is $\omega^{+}=4 d \xi^{i} \wedge d \zeta^{i}$. We study a compact group with $k$ generators acting on $M$ by some antihermitian matrix representation $\left(T_{A}\right)_{i j}, A=1, \ldots, k$, with

$$
k_{A}=-\left(T_{A}\right)_{j i}\left(\zeta^{i} \frac{\partial}{\partial \zeta^{j}}-\xi^{j} \frac{\partial}{\partial \xi^{i}}-\bar{\zeta}^{j} \frac{\partial}{\partial \bar{\zeta}^{i}}+\bar{\xi}^{i} \frac{\partial}{\partial \bar{\xi}^{j}}\right) .
$$

This defines a triholomorphic action. The Killing potentials for the Killing vectors are

$$
\begin{gathered}
\mu_{A}^{(1)}=-i \bar{\zeta}^{i}\left(T_{A}\right)_{i j} \zeta^{j}+i \xi^{i}\left(T_{A}\right)_{i j} \xi^{j}+c_{A}, \\
\mu_{A}^{+}=-2\left(\xi^{i}\left(T_{A}\right)_{i j} \zeta^{j}+b_{A}\right),
\end{gathered}
$$

where $c_{A}=\bar{c}_{A}$ and $b_{A}, \bar{b}_{A}$ are arbitrary constants for each $U(1)$ factor. The Kähler potential for $M$ is

$$
K=\bar{\zeta}^{i} \zeta^{i}+\xi^{i} \bar{\zeta}^{i},
$$

whereas the Kähler potential for the quotient manifold is [cf. (2.23)]

$$
\hat{K}=\bar{\zeta}^{i}\left(e^{i V}\right)_{i j} \zeta^{j}+\xi^{i}\left(e^{-i V}\right)_{i j} \bar{\zeta}^{j}-c_{A} V^{A},
$$

where $V=V^{A} T_{A}$ is determined by the algebraic equations

$$
i \bar{\zeta} e^{i V} T_{A} \zeta-i \xi T_{A} e^{-i V \bar{\xi}}-c_{A}=0 .
$$

The holomorphic subspace (2.17) is the quadric

$$
\xi T_{A} \zeta+b_{A}=0 .
$$

\section{(C) Quotients and Legendre Transforms}

In this subsection we apply the quotient construction to hyperkähler manifolds obtained by the Legendre transform method; such ( $4 n$ dimensional) manifolds have $n$ commuting triholomorphic isometries, and thus we can divide by $k<n U(1)$ factors to get a $4(n-k)$ dimensional manifold. In the special nonholomorphic coordinates $x, y, z, \bar{z}$ [cf. (2.4-7)] Eqs. (2.17-19) that define the hyperkähler quotient become linear, and the construction can be carried out explicitly.

We start from $K\left(u^{i}+\bar{u}^{i}, z^{i}, \bar{z}^{i}\right)$ as defined in (2.4) and divide by a linear combination of $k$ of the isometries (2.9):

$$
\begin{aligned}
& k_{A}^{i} \partial_{i}=i q_{A}^{i}\left(\frac{\partial}{\partial u^{i}}-\frac{\partial}{\partial \bar{u}^{i}}\right) \quad \begin{array}{l}
i=1, \ldots, 4 n \\
i=1, \ldots, n
\end{array} \\
& A=1, \ldots, k<n \text {. }
\end{aligned}
$$

From (1.10) we calculate

$$
\mu_{A}^{(1)}=-q_{A}^{i} \frac{\partial K}{\partial u^{i}}-c_{A}, \quad c_{A}=\bar{c}_{A}
$$

and from (1.15), (2.8):

$$
\mu_{A}^{+}=2\left(i q_{A}^{i} z^{i}+b_{A}\right)
$$


Evaluating (2.23) we then find

$$
\hat{K}(u, \bar{u}, z, \bar{z}, V)=c_{A} V^{A}+K\left(u^{i}+\bar{u}^{i}+V^{i}, z^{i}, \bar{z}^{i}\right),
$$

where $V^{A}$ are the auxiliary real functions and $V^{i}=q_{A}^{i} V^{A}$. Now (2.4) gives

$$
\widehat{K}=c_{A} V^{A}+F(x, z, \bar{z})-\left(u^{i}+\bar{u}^{i}+V^{i}\right) x^{i} .
$$

The quotient $(2.17,22)$ reduces to restricting $(x, z, \bar{z})$ to the hyperplanes

$$
\begin{gathered}
\mu_{A}^{(1)}=q_{A}^{i} x^{i}-c_{A}=0, \\
\frac{1}{2} \mu_{A}^{+}=i q_{A}^{i} z^{i}+b_{A}=0 .
\end{gathered}
$$

The relation (2.37a) makes $\hat{K}$ [cf. (2.36)] independent of $V$. Since $x, z$, and $\bar{z}$ are inert under the isometry, this defines the quotient. The examples given at the end of Sect. 2.A arise as quotients of flat space in its Legendre transform description, with the constants $c_{A}, b_{A}$, and $\bar{b}_{A}$ assembled into the vector $\vec{\varrho}_{A}(2.15)$.

\section{Geometric Interpretation}

\section{(A) Quotient Manifolds}

If $X$ is a topological space with an action of a group $G$, then the quotient space $X / G$ - the space of orbits - has a natural topology with respect to which the projection map

$$
p: X \rightarrow X / G
$$

is continuous. Bad actions of groups, such as the irrational flow on a torus, can give non-Hausdorff quotient spaces, but if $G$ is compact and $X$ is Hausdorff, then so is $X / G$.

Suppose now we replace $X$ by a manifold $M$ and assume that $G$ is a compact Lie group acting smoothly on $M$. If $G$ acts freely, i.e., the identity element is the only one with fixed points, then the quotient space $M / G$ can be given the structure of a manifold with respect to which the projection $p$ is a smooth submersion. Because the action is free, non-zero vector fields generated by the Lie algebra $g$ have no zeroes and so through each point $m \in M$ there is a subspace $V_{m}$ (with $\operatorname{dim} V_{m}=\operatorname{dim} \mathfrak{g}$ ) of the tangent space $T_{m}$ spanned by the vector fields in $\mathfrak{g}$. This space, called the vertical space, is the tangent space to the orbit of $G$ through $m$. The tangent space to $p(m) \in M / G$ is then isomorphic to the quotient vector space $T_{m} / V_{m}$ (see Fig. 2).

Now let $M$ be given a Riemannian metric $g$, and suppose $G$ acts as isometries. [In this case the vector fields $k_{A}$ in (1.6) form a basis for $V_{m}$.] We may define an induced Riemannian metric on $M / G$ as follows. Let $H_{m} \subset T_{m}$ be the subspace of vectors orthogonal to $V_{m}$, called the horizontal space. Then the derivative of $p$ maps $H_{m}$ isomorphically to the tangent space of the quotient at $p(m)$. A tangent vector $X \in T_{p(m)}$ then has a unique horizontal lift $\tilde{X} \in H_{m} \subset T_{m}$, and we define an inner product $h$ on $T_{p(m)}$ by

$$
h(X, Y)=g(\tilde{X}, \tilde{Y}) .
$$




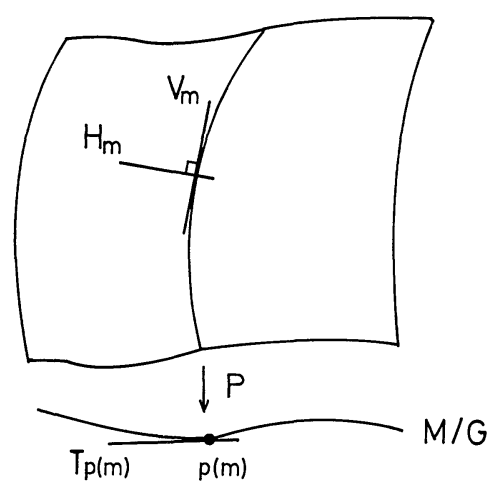

Fig. 2. The total space $M$ and its projection to the quotient space $M / G$

Since $G$ preserves the metric $g$, this is independent of the choice of point $m$ in the orbit $p^{-1}(p(m))$.

This family of horizontal subspaces has an interpretation in terms of connections. The manifold $M$ is, by definition of the free action of $G$, a principal $G$-bundle over $M / G$. Also, the vector fields corresponding to a basis of the Lie algebra $g$ form a basis for $V_{m}$ at each point $m \in M$, hence the orthogonal projection from $T_{m}$ to $V_{m}$ defines a 1 -form $\theta$ with values in $g$ and transforming under the adjoint representation of $G$. This is therefore a connection form for the principal bundle (see [2]).

In more concrete terms, if $k_{A}(1 \leqq A \leqq \operatorname{dim} G)$ are the vector fields corresponding to a basis of $\mathfrak{g}$, and $x^{i}$ are local coordinates on $M$,

$$
\theta^{A}\left(\frac{\partial}{\partial x^{i}}\right)=g\left(k_{B}, \frac{\partial}{\partial x^{i}}\right) H^{A B},
$$

where $H^{A B} \equiv\left[g\left(k_{A}, k_{B}\right)\right]^{-1}$. Given a local gauge, i.e., section $s$ of $p: M \rightarrow M / G$, the usual connection form is

$$
A_{i}^{A} d y^{i}=s^{*}\left(\theta_{i}^{A} d x^{i}\right)
$$

The pullback of $A_{i}^{A}$ to spacetime is the connection in (4.16).

Now suppose $M$ is endowed with some extra differential geometric structure compatible with the metric, then $M / G$ will not in general inherit the same structure. For example if $M$ is a Kähler manifold and $G=U(1)$, then $M / G$ is odd-dimensional and never Kählerian. There is nevertheless a means of obtaining one Kähler manifold from another with a group action, which generalizes in a straightforward way to the hyperkähler case. This is based on the symplectic quotient or reduced phase space of Marsden and Weinstein [12].

\section{(B) Symplectic Quotients}

Let $M^{2 n}$ be a symplectic manifold, i.e., a manifold with a closed, non-degenerate 2-form $\omega$. Suppose also that the Lie group $G$ acts on $M$ preserving the symplectic form. Then, if $X$ is a vector field generated by this action, the Lie derivative $L_{X} \omega$ vanishes. 
Now for any differential form $\omega$ of degree $p$

$$
L_{X} \omega=i(X) d \omega+d(i(X) \omega),
$$

where $i(X) \omega$ denotes the $(p-1)$-form obtained by taking the interior product (contraction) with $X$. We therefore have, for the symplectic 2 -form $\omega$,

$$
0=L_{X} \omega=d(i(X) \omega),
$$

hence the 1 -form $i(X) \omega$ is closed, and if the first cohomology group $H^{1}(M, R)$ vanishes, then by de Rham's theorem there is a function $\mu^{X}$ on $M$ such that [cf. (1.9)]

$$
d \mu^{X}=i(X) \omega .
$$

(Locally, this is always true, but we require $\mu^{X}$ to exist globally.) Since $\omega$ is nondegenerate, the vector field $X$ is equally determined by the 1 -form $i(X) \omega$, and hence by the Hamiltonian function $\mu^{X}$. Adding a constant to $\mu^{X}$ clearly leaves (3.7) unchanged and this (if $M$ is connected) is the only ambiguity in the choice of $\mu^{X}$.

Putting all these functions together, we obtain a map

$$
\mu: M \rightarrow \mathrm{g}^{*}
$$

to the dual space $\mathrm{g}^{*}$ of the Lie algebra defined by

$$
\langle\mu(m), \xi\rangle=\mu^{X}(m),
$$

where $X$ is the vector field generated by $\xi \in \mathfrak{g}$.

There is a natural action of $G$ both on $M$ and $\mathrm{g}^{*}$ and we should like to adjust the ambiguity in the definition of $\mu$ to make $\mu$ equivariant, i.e., commute with the two actions [cf.(1.11)]. For a general Lie group $G$ there may be an obstruction to doing this which lies in a cohomology group of the Lie algebra, but if $G$ is semi-simple or a torus this obstruction vanishes [12]. In any case, if $G$ is compact we may assume that $\mu$ is equivariant.

When an equivariant function $\mu$ exists, it is called a moment map for the symplectic action of $G$ on $M$. The remaining ambiguity in the choice of $\mu$ is now simply the addition of a constant element in $\mathrm{g}^{*}$ which is left fixed by $G$, i.e., a character of $G$. If we have an invariant inner product on $\mathfrak{g}$, then identifying $\mathfrak{g}^{*} \cong \mathfrak{g}$ we are allowed to modify $\mu$ by adding a constant in the center of $\mathfrak{g}$.

The nomenclature is clear if we take $M=T^{*} R^{3}$, the cotangent bundle of $R^{3}$ with its canonical symplectic structure

$$
\frac{1}{2} \omega=d x^{1} \wedge d p^{1}+d x^{2} \wedge d p^{2}+d x^{3} \wedge d p^{3} .
$$

A vector field $X=a^{i} \partial / \partial x^{i}$ corresponding to a translation satisfies

$$
i(X) \omega=a^{i} d p^{i}=d\left(a^{i} p^{i}\right) .
$$

Hence $\mu: T^{*} R^{3} \rightarrow \mathrm{g}^{*}=R^{3}$ is

$$
\mu(\vec{x}, \vec{p})=\vec{p},
$$

giving the linear momentum. 
For the natural action of $\mathrm{SO}(3)$, where $\mathrm{g}^{*} \cong R^{3}$, we obtain

$$
\mu(\vec{x}, \vec{p})=\vec{x} \times \vec{p}
$$

giving the angular momentum.

Consider now the submanifold $N=\mu^{-1}(\mu(m))$ (the points in $M$ that map into the same element of $\mathrm{g}^{*}$ ) and let $Y$ be a tangent vector to $N$. Then

$$
d \mu(Y)=0,
$$

and hence

$$
0=d \mu^{X}(Y)=\omega(X, Y)
$$

for all vector fields $X$ generated by $\mathfrak{g}$. As the form $\omega$ is non-degenerate this gives $\operatorname{dim} G$ independent equations for $Y$ giving

$$
\operatorname{dim} \operatorname{Ker} d \mu=2 n-\operatorname{dim} G
$$

so $N$ is a submanifold of dimension $2 n-\operatorname{dim} G$.

Now suppose there exists $m \in M$ such that $\mu(m)=0$, and let $N=\mu^{-1}(0)$. Since $G$ certainly keeps the origin in $\mathrm{g}^{*}$ fixed, and $\mu$ is equivariant, $G$ acts on the manifold $N$. We form the quotient $N / G$ which is a manifold of dimension $2 n-2 \operatorname{dim} G$. It posesses a natural 2-form $\varrho$ defined by

$$
\varrho\left(Y_{1}, Y_{2}\right)=\omega\left(\tilde{Y}_{1}, \tilde{Y}_{2}\right),
$$

where $\tilde{Y}_{i}$ is any tangent vector to $N$ which projects to $Y_{i}$, a tangent vector to $N / G$. We must check that this is well-defined. However, if $X$ is a vector field generated by $\mathfrak{g}$, then $\omega\left(X+\widetilde{Y}_{1}, \widetilde{Y}_{2}\right)=\omega\left(X, \widetilde{Y}_{2}\right)+\omega\left(\widetilde{Y}_{1}, \widetilde{Y}_{2}\right)=\omega\left(\widetilde{Y}_{1}, \widetilde{Y}_{2}\right)$ from (3.15).

The form $\varrho$ is in fact a symplectic form on $N / G$. We first check its nondegeneracy.

If $\varrho\left(Y_{1}, Y\right)=0$ for all $Y$, then by the definition of $\varrho, \omega\left(\widetilde{Y}_{1}, \widetilde{Y}\right)=0$ for all $\tilde{Y}$ tangent to $N$. But from (3.15), the only vectors which annihilate the $(2 n-\operatorname{dim} G)$ dimensional tangent space to $N$ at $m$ are those generated by $g$, hence $\widetilde{Y}_{1}$ is the restriction at $m$ of some vector field $X$ from $\mathfrak{g}$, so that $Y_{1}$, the projection of $\widetilde{Y}_{1}$, to $N / G$, is zero. Thus $\varrho$ is non-degenerate.

Secondly we prove that $\varrho$ is closed. If we let $j: N \rightarrow M$ denote the inclusion map, then by the definition (3.17),

$$
p^{*} \varrho=j^{*} \omega,
$$

where $p: N \rightarrow N / G$ is the projection. Applying the exterior derivative,

$$
p^{*}(d \varrho)=d\left(p^{*} \varrho\right)=d\left(j^{*} \omega\right)=j^{*}(d \omega)=0 .
$$

Since $p$ is a submersion ( $p^{*}$ is injective on forms), the 3-form $d \varrho$ on $N / G$ is itself zero, so $\varrho$ is closed.

We have thus produced a "quotient" of one symplectic manifold by a group of symplectic transformations to obtain another whose dimension is $2 \operatorname{dim} G$ less. More generally, if we take a point $x \in \mathfrak{g}^{*}$ which is not fixed by $G$, but has isotropy subgroup $H$, then $\mu^{-1}(x) / H$ has a symplectic structure in the same way. 


\section{(C) Kähler Quotients}

Let $M^{2 n}$ now be a Kähler manifold, i.e., a complex manifold whose complex structure I is covariantly constant with respect to the Levi-Civita connection of a metric $g$ invariant under I (i.e., a hermitian metric). Under these circumstances

$$
\omega(X, Y)=g(\mathbf{I} X, Y)
$$

defines a closed (in fact covariantly constant), non-degenerate 2-form and so $M$ is in particular a symplectic manifold. The form $\omega$ is called the fundamental 2-form or Kähler form.

Suppose $G$ is a compact Lie group acting freely on $M$ and preserving both the metric and the symplectic form [and hence by (3.20) the complex structure] [cf. (1.4)]. We can produce, as above, the symplectic quotient $\hat{M}$, which is a symplectic manifold. It also has a naturally induced metric since $\hat{M}=N / G$, where $N \subset M$ is endowed with the metric $g$ restricted to $N$, and in Sect. $3 \mathrm{~A}$ we saw how to put a metric on a quotient. We show:

Theorem 3.1. The induced metric on $\hat{M}$ is Kählerian, with Kähler form @.

Proof. We consider first $N=\mu^{-1}(0) \subset M$ with its induced metric. The construction of the Levi-Civita connection on $N$ is standard in differential geometry [2]: Take a tangent vector field $Y$ on $N$. This is a section of $T M$ restricted to $N$. Using the LeviCivita connection on $T M$ of the metric $g$ we take its covariant derivative. This will in general no longer lie in the subbundle $\left.T N \subset T M\right|_{N}$ but by orthogonal projection we push it back into $T N$ and obtain a connection on $T N$ which is the Levi-Civita connection. All that is required is to check that it preserves the metric and is torsion-free, which is straightforward.

Finding the Levi-Civita connection of the quotient metric on $N / G$ is similar, but less familiar. Here we have the horizontal bundle $H \subset T N$ over $N$ which is identified by the projection $p: N \rightarrow N / G$ with $T(N / G)$. The Levi-Civita connection on $T(N / G)$ with the metric $h$ defined in Sect. 3A then pulls back to a $G$-invariant connection on $H$ over $N$. This, we claim, is the connection $\nabla^{H}$ on $H$ obtained by orthogonal projection of the Levi-Civita connection on $T N$. To prove this we need to check that $\nabla^{H}$ preserves the metric $h$ on $H$ (which is clear) and is torsion-free for horizontal vector fields. So let $X, Y$ be two commuting vector fields on $N / G$. Lift them to horizontal fields $\tilde{X}$, $\widetilde{Y}$ on $N$. They no longer commute, but from the principal bundle formalism of connections [2], their Lie bracket is vertical. In fact

$$
[\tilde{X}, \tilde{Y}]=F(X, Y) \text {, }
$$

where $F$ is the curvature of the connection $\theta$ defined in Sect. 3A by the horizontal subspaces. Consequently,

$$
\nabla_{X}^{H} Y-\nabla_{Y}^{H} X=P_{H}\left(\nabla_{\tilde{X}} \tilde{Y}-\nabla_{\tilde{Y}} \tilde{X}\right)=P_{H}(F(X, Y)),
$$

where $P_{H}$ is the projection onto $H$.

Putting these two constructions together we see that the Levi-Civita connection of $\hat{M}=N / G$ may be described by the orthogonal projection of the Levi-Civita connection of $M$ from $T M$ over $N$ to the horizontal subbundle $H$. 
Consider, however, the orthogonal complement of $H$ in $T M$. The complement of $T N$ in $T M$ is spanned at $m \in N$ by the normal vectors $\operatorname{grad} \mu_{A}, A=1, \ldots, \operatorname{dim} G$ and the complement of $H$ in $T N$ is spanned by the vertical vectors $k_{A}$, where the vector fields $k_{A}$ arise from a basis of $\mathfrak{g}$. But

$$
g\left(\operatorname{grad} \mu^{X}, Y\right)=d \mu^{X}(Y)=\omega(X, Y)=g(\mathbf{I} X, Y),
$$

and so

$$
\operatorname{grad} \mu^{X}=\mathbf{I} X \text {. }
$$

Thus the vector space spanned by grad $\mu_{A}$ and $k_{A}$ is a complex vector space, and so the complement of $H$ and hence $H$ itself is a complex vector bundle.

The metric on $M$ was Kählerian, so I commutes with the covariant derivative. Since $H$ is complex, I commutes with the orthogonal projection and so I commutes with the connection $\nabla^{H}$. Thus the induced metric on $\hat{M}$ is Kählerian.

This infinitesimal calculation disguises one essential aspect of the problem: the vector fields $X, \mathbf{I} X$ generate a complex Lie algebra of holomorphic vector fields and hence a local holomorphic action of the complex group $G^{C}$ obtained from the complex Lie algebra $g \otimes C$. Suppose this extends to a global action (equivalent to the completeness of the vector fields $I X$ ) then the symplectic quotient $\hat{M}$, considered as a complex manifold, is simply the ordinary manifold quotient as in Sect. 3A in the holomorphic setting. However, since $G^{C}$ is not compact, we would obtain non-Hausdorff behaviour in this quotient, unless we restrict the action of $G^{C}$ to a suitable open set of points in $\hat{M}$. This open set consists of those points whose $G^{C}$ orbits meet $N$ and are called stable points (see Fig. 3). In many cases in algebraic geometry [13] this idea of stability coincides with a pre-existing algebraic definition called Mumford stability.

\section{(D) Hyperkähler Quotients}

Suppose finally that $M^{4 n}$ is a hyperkähler manifold having a metric $g$ and covariantly constant complex structures $\mathbf{I}, \mathbf{J}, \mathbf{K}$ which behave algebraically like quaternions:

$$
\mathbf{I}^{2}=\mathbf{J}^{2}=\mathbf{K}^{2}=-1, \quad \mathbf{I} \mathbf{J}=-\mathbf{J} \mathbf{I}=\mathbf{K} \quad \text { etc. }
$$

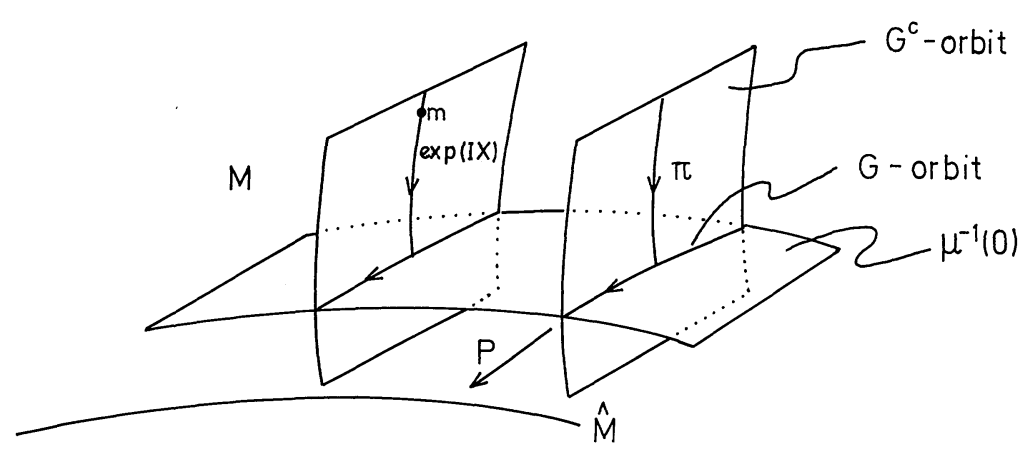

Fig. 3. The orbits of the group $G$ and of its complexification $G^{C}$. $G$ acts on $\mu^{-1}(0)$ and $\hat{M}$ is the quotient space corresponding to this action. The same space is obtained if one considers the extension of $\mu^{-1}(0)$ by $\exp (I X)$ and takes the quotient by $G^{C}$ 
Let $G$ be a compact Lie group of isometries acting freely on $M$ and preserving the structures $\mathbf{I}, \mathbf{J}, \mathbf{K}$. The group $G$ preserves the three Kähler forms $\omega_{1}, \omega_{2}, \omega_{3}$ corresponding to the three complex structures, so we may define three moment maps $\mu_{1}, \mu_{2}, \mu_{3}$. More invariantly these can be written as a single map

$$
\mu: M \rightarrow \mathrm{g}^{*} \otimes R^{3} .
$$

The basic theorem, generalizing Theorem 3.1 is the following:

Theorem 3.2. The quotient metric on $\mu^{-1}(0) / G$ is hyperkählerian.

Proof. We first focus attention on one complex structure I, with Kähler form $\omega_{1}$. Consider the complex function

$$
\mu_{+}=\mu_{2}+i \mu_{3}: M \rightarrow \mathrm{g}^{*} \otimes C .
$$

Now

$$
d \mu_{+}^{X}(Y)=\omega_{2}(X, Y)+i \omega_{3}(X, Y)=g(\mathbf{J} X, Y)+i g(\mathbf{K} X, Y),
$$

and

$$
d \mu_{+}^{X}(\mathbf{I} Y)=g(\mathbf{J} X, \mathbf{I} Y)+i g(\mathbf{K} X, \mathbf{I} Y)=-g(\mathbf{K} X, Y)+i g(\mathbf{J} X, Y)
$$

Thus

$$
d \mu_{+}^{X}(\mathbf{I} Y)=i d \mu_{+}^{X}(Y)
$$

for each vector field $Y$.

Let $\partial / \partial \bar{z}^{p}$ be a basic complex vector field arising from a local coordinate system $\left\{z^{p}\right\}$, holomorphic with respect to I, on $M$. Then

$$
\mathbf{I} \frac{\partial}{\partial \bar{z}^{p}}=-i \frac{\partial}{\partial \bar{z}^{p}}
$$

and so from $(3.30,31)$

$$
i \frac{\partial \mu_{+}^{X}}{\partial \bar{z}^{p}}=d \mu_{+}^{X}\left(\mathbf{I} \frac{\partial}{\partial \bar{z}^{p}}\right)=-i \frac{\partial \mu_{+}^{X}}{\partial \bar{z}^{p}},
$$

and hence $\mu_{+}^{X}$ is a holomorphic function.

Thus $N=\mu_{+}^{-1}(0)=\mu_{2}^{-1}(0) \cap \mu_{3}^{-1}(0)$ is a complex submanifold of $M$, with respect to the complex structure $\mathbf{I}$, and so its induced metric is Kählerian.

The group $G$ acts on $N$ preserving the Kähler form and its moment map is clearly the restriction of $\mu_{1}$ to $N$. Hence by Theorem 3.1 the quotient metric on $N \cap \mu_{1}^{-1}(0) / G=\mu^{-1}(0) / G$ is Kählerian with respect to the complex structure $\mathbf{I}$.

To complete the proof, repeat the argument with the complex structures $\mathbf{J}$ and K. 2-form

To interpret the function $\mu_{+}$which arose in the proof, consider the complex

$$
\omega_{+}=\omega_{2}+i \omega_{3} .
$$


Then

$$
\begin{aligned}
\omega_{+}\left(\frac{\partial}{\partial \bar{z}^{p}}, Y\right) & =g\left(\mathbf{J} \frac{\partial}{\partial \bar{z}^{p}}, Y\right)+i g\left(\mathbf{K} \frac{\partial}{\partial \bar{z}^{p}}, Y\right) \\
& =i g\left(\mathbf{J I} \frac{\partial}{\partial \bar{z}^{p}}, Y\right)+i g\left(\mathbf{K} \frac{\partial}{\partial \bar{z}^{p}}, Y\right) \\
& =-i g\left(\mathbf{K} \frac{\partial}{\partial \bar{z}^{p}}, Y\right)+i g\left(\mathbf{K} \frac{\partial}{\partial \bar{z}^{p}}, Y\right)=0 .
\end{aligned}
$$

Thus $\omega_{+}$is a 2 -form of type $(2,0)$, i.e., in local coordinates

$$
\omega_{+}=\omega_{p q} d z^{p} \wedge d z^{q} .
$$

Since it is covariantly constant it is holomorphic and easily seen to be nondegenerate in the complex sense:

$$
\omega_{+}^{n} \neq 0 \text {. }
$$

The action of $G^{C}$ on $M$ preserves $\omega_{+}$and the function

$$
\mu_{+}: M \rightarrow \mathfrak{g}^{*} \otimes C
$$

is the complex moment map for this action. Thus the hyperkähler quotient $\mu^{-1}(0) / G$ is the symplectic quotient of Sect. 3B but in a holomorphic setting.

The procedure for finding new examples of hyperkähler manifolds in Sect. 2 involves taking $M$ to be a flat hyperkähler manifold - a vector space over the quaternions - and considering the actions of groups on this. In particular any subgroup $G \leqq \operatorname{Sp}(k)$ may be taken. The group requires a center to produce a moment map for which the origin in the vector space (which is clearly not acted on freely by $G$ ) is not contained in $\mu^{-1}(0)$.

\section{(E) Kähler Potentials}

For the relationship with the supersymmetric $\sigma$-model, it is important to find the Kähler potential $\hat{K}$ of the quotient space $\hat{M}$ of Sect. $3 C$, i.e., a real function such that

$$
\varrho=2 i \partial \bar{\partial} \hat{K},
$$

where $\varrho$ is the Kähler form of $\hat{M}$. The function $\hat{K}$ is well-defined modulo the addition of a function $f+\bar{f}$, where $f$ is an arbitrary holomorphic function. The geometrical interpretation of the Kähler potential is via connections on complex line bundles.

Let $M$ be a Kähler manifold with Kähler form $\omega$, and suppose $\omega$ represents an integral class in the de Rham cohomology group $H^{2}(M, R)$. We may then interpret $2 \pi i \omega$ as the curvature form of a connection on a hermitian line bundle $L$ over $M$. Moreover, since $\omega$ is of type $(1,1)$ we can take this to be a holomorphic line bundle.

Remark. The condition that the cohomology class of the Kähler form be integral is not strictly necessary: One can interpret $\omega$ as the curvature form of a connection on a principal bundle with the translation group $R$ (rather than its quotient $U(1)=R / Z)$. However, connections on vector bundles are more familiar than 
connections on affine bundles, and so we use this language below [the affine description is used in Sect. 6, Eqs. (6.50-53)].

Let $s$ be a local non-vanishing holomorphic section of the line bundle $L$; then we define the connection by

$$
\nabla s=\frac{\partial\|s\|^{2}}{\|s\|} s=\theta s .
$$

This is the unique connection compatible with (i.e., preserving) both the holomorphic structure and the hermitian metric $\|s\|^{2}=h s \bar{s}$. The curvature $F$ of the connection is

$$
F=d \theta=d \partial \log \|s\|^{2}=-\partial \bar{\partial} \log \|s\|^{2}
$$

Thus

$$
\omega=\frac{i}{2 \pi} \partial \bar{\partial} \log \|s\|^{2}
$$

giving $K=\frac{1}{4 \pi} \log \|s\|^{2}$ as a Kähler potential. Changing $K$ by adding a function $f+\bar{f}$ corresponds to changing the local holomorphic section $s$.

Now suppose we have a compact group $G$ acting as in Sect. 3C, preserving the metric and complex structure and with a moment map

$$
\mu: M \rightarrow \mathrm{g}^{*} .
$$

Under these circumstances we obtain an action of the Lie algebra of $G$, and of $G$ itself under suitable completeness conditions on the line bundle $L$. We define the Lie derivative of a section $s$ of $L$ by a vector field $X$ generated by $g$ according to the rule [12]

$$
L_{X} s=\nabla_{X} s+4 \pi i \mu^{X} s \equiv 4 \pi v^{X} s,
$$

where $v^{X}$ is a holomorphic function. To see that this is an action, note that

$$
\left[L_{X}, L_{Y}\right]=\left[\nabla_{X}+4 \pi i \mu^{X}, \nabla_{Y}+4 \pi i \mu^{Y}\right]=\left[\nabla_{X}, \nabla_{Y}\right]+4 \pi i\left(X \mu^{Y}-Y \mu^{X}\right) .
$$

But the curvature $F$ of the connection $\nabla$ gives

$$
4 \pi i \omega(X, Y)=2 F(X, Y)=\nabla_{X} \nabla_{Y}-\nabla_{Y} \nabla_{X}-\nabla_{[X, Y]},
$$

hence,

$$
\left[L_{X}, L_{Y}\right]=\nabla_{[X, Y]}+4 \pi i \omega(X, Y)+4 \pi i\left(X \mu^{Y}-Y \mu^{X}\right) .
$$

On the other hand, by the equivariance of the moment map,

$$
X \mu^{Y}=\mu^{[X, Y]},
$$

and by the definition of $\mu^{X}$,

$$
Y \mu^{X}=d \mu^{X}(Y)=\omega(X, Y)
$$

Thus from (3.46) we obtain

$$
\left[L_{X}, L_{Y}\right]=\nabla_{[X, Y]}+4 \pi i \mu^{[X, Y]}=L_{[X, Y]},
$$


showing the existence of an action of the Lie algebra. In a similar manner we can check that this action extends to an action of $g \otimes C$ by setting

$$
L_{\mathbf{I} X} s=\nabla_{\mathbf{I} X} s-4 \pi \mu^{X} s .
$$

Note that from (3.50), the vector fields $\mathbf{I} X$ do not preserve the hermitian form $h$ on $L$. In fact, since the connection $\nabla$ does preserve it, it follows directly from (3.50) that

$$
L_{\mathbf{I X}} h=8 \pi \mu^{X} h .
$$

Returning to the quotient construction of a Kähler manifold in Sect. 3C we have the diagram in Fig. 3. The holomorphic map from a point $m \in M$ to $\widehat{M}=\mu^{-1}(0) / G$ is obtained by first taking $m$ to $\mu^{-1}(0)$ by a group element of the form $\exp (\mathbf{I} X) \in G^{C}$ and then assigning to it the $G$-orbit in $\mu^{-1}(0)$ in which it lies. The holomorphic map therefore factors as

$$
M \stackrel{\pi}{\longrightarrow} \mu^{-1}(0) \stackrel{p}{\longrightarrow} \mu^{-1}(0) / G
$$

with

$$
\pi(m)=\exp (\mathbf{I} X(m)) \cdot m .
$$

The Kähler form $\varrho$ of $\hat{M}$ satisfies, from Sect. 3B,

$$
p^{*} \varrho=j^{*} \omega
$$

where $j: \mu^{-1}(0) \rightarrow M$ is the inclusion, so pulling back $\varrho$ from $\hat{M}$ to $M$ is the same as restricting $\omega$ to $\mu^{-1}(0)$ and pulling back by $\pi$. Call this form $\hat{\omega}$.

To find the corresponding Kähler potential, pulled back to $M$, we use the curvature interpretation of the symplectic form. Then, parallel to the description of $\omega$ above, we see that $2 \pi i \hat{\omega}$ is the curvature form of the connection $\hat{\nabla}$ obtained by restricting to $\mu^{-1}(0)$ and pulling back to $M$ via the projection $\pi$. Since the fibers of $\pi$ are contained in $G^{C}$ orbits and $L$ is acted on by $G^{C}$, the connection $\hat{\nabla}$ is defined on the same line bundle $L$.

The hermitian metric which defines the Kähler potential is left invariant by $G$ but not by $G^{C}$. In fact if $h$ denotes the hermitian metric on $L$ and $g \in G^{C}$, we may define a function $c(m, g)$ on $M \times G^{C}$ by exponentiating (3.51)

$$
\left(g^{*} h\right)(m)=e^{c(m, g)} h(m) .
$$

Now if $s$ is a local non-vanishing holomorphic section of $L$ on the quotient $\hat{M}$, the Kähler potential is

$$
\hat{K}=\frac{1}{4 \pi} \log \hat{h} s \bar{s},
$$

where $\hat{h}$ is the hermitian metric preserved by $\hat{\nabla}$. But this, by the description of the connection above is

$$
\widehat{h}(m)=\left(g^{*} h\right)(m),
$$

where $g=\exp (\mathbf{I} X)$ is the element of $G^{C}$ which takes $m$ to $\mu^{-1}(0)$. Hence from (3.55),

$$
\widehat{K}-K=\frac{1}{4 \pi} \log \left(g^{*} h / h\right)=\frac{1}{4 \pi} c(m, g),
$$


so

$$
\hat{K}=K+\frac{1}{4 \pi} c(m, g)
$$

where $g=g(m)$ is defined as above. Note that, from (3.55), $c$ satisfies the condition

$$
c\left(g_{1} m, g_{2}\right)+c\left(m, g_{1}\right)=c\left(m, g_{2} g_{1}\right)
$$

and hence

$$
c\left(e^{i X} m, e^{i Y}\right)+c\left(m, e^{i X}\right)=c\left(m, e^{i Y} e^{i X}\right) .
$$

Differentiating with respect to $Y$ and setting $Y=0$ we see that the derivative of $c(m, g)$ with respect to the second variable, in the direction $Y$ is equal to the derivative of $c\left(g \cdot m, e^{i Y}\right)$ in the direction $Y$ and from (3.51) and (3.55) this is $8 \pi \mu^{Y}(g \cdot m)$. Thus the choice of $g(m)$ to make $\mu(g \cdot m)=0$ may be interpreted as making the function $c(m, g)$ independent of the second variable, as discussed in (2.24). The formal expression for $c(m, g)$ in (2.23) is essentially the integration of (3.51)-(3.55).

Calculating the Kähler potential thus involves solving the equation

$$
\mu\left(e^{i X} \cdot m\right)=0
$$

for $X$.

In the hyperkähler case this is all carried out on the Kähler manifold $\mu_{+}^{-1}(0)$. There is, however, one special case in the realm of hyperkähler geometry where the Kähler potential arises in a simpler manner. Suppose $M^{4 n}$ is a hyperkähler manifold with an isometric action of the circle which acts non-trivially on the 3 -dimensional space of covariant constant 2-forms spanned by $\omega_{1}, \omega_{2}, \omega_{3}$. There is only one non-trivial action, i.e., the circle fixes $\omega_{1}$ and rotates the $\omega_{2}, \omega_{3}$ plane. If $X$ is the corresponding Killing field then

$$
L_{X} \omega_{1}=0, \quad L_{X} \omega_{2}=\omega_{3}, \quad L_{X} \omega_{3}=-\omega_{2} .
$$

Now let $\mu$ be the moment map of this action with respect to the preserved symplectic form $\omega_{1}$. Consider the complex structure $\mathbf{J}$ with Kähler form $\omega_{2}$. Now,

$$
d \mu(\mathbf{J} Y)=(\partial \mu+\bar{\partial} \mu)(\mathbf{J} Y)=i(\partial \mu-\bar{\partial} \mu)(Y),
$$

where $\partial$ and $\bar{\partial}$ are the $(1,0)$ and $(0,1)$ parts of $d$ with respect to $\mathbf{J}$. But

$$
d \mu(\mathbf{J} Y)=i(X) \omega(\mathbf{J} Y)=g(\mathbf{I} X, \mathbf{J} Y)=g(\mathbf{K} X, Y)=\omega_{3}(X, Y) .
$$

Thus from (3.64),

$$
i(X) \omega_{3}=i(\partial \mu-\bar{\partial} \mu)
$$

Hence

$$
-2 i \partial \bar{\partial} \mu=d\left(i(X) \omega_{3}\right)=L_{X} \omega_{3}=-\omega_{2},
$$

i.e.,

$$
\omega_{2}=2 i \partial \bar{\partial} \mu
$$


Hence the moment map for $\omega_{1}$ is a Kähler potential for $\omega_{2}$. This is a situation which arises quite frequently in examples, such as the Taub-NUT metric, EguchiHanson metric, and Calabi metric.

\section{(F) Twistor Spaces}

The Legendre transform construction of Sect. 2 produces hyperkähler metrics from holomorphic functions. The natural setting for this is a generalization of Penrose's non-linear graviton construction [14] to hyperkähler manifolds, which we describe here. This itself forms part of a theory of quaternionic manifolds developed by Salamon $[15,16]$.

Let $M^{4 n}$ be a hyperkähler manifold with complex structures $\mathbf{I}, \mathbf{J}, \mathbf{K}$. Then if $(a, b, c)$ is a unit vector in $R^{3}$, the multiplicative properties of $\mathbf{I}, \mathbf{J}$, and $\mathbf{K}$ imply

$$
(a \mathbf{I}+b \mathbf{J}+c \mathbf{K})^{2}=-1,
$$

and so there is a whole 2-sphere of complex structures compatible with the metric and connection. The idea of the twistor approach is to incorporate all these structures into one complex structure on a larger manifold, the twistor space of $M$. In turn, its holomorphic structure contains all the metric information of the hyperkähler manifold. In the case of the Legendre transform this structure depends on a single holomorphic function of $(n+1)$ variables.

We begin by considering the complex structure of the Riemann sphere $S^{2}$, considered as the complex projective line $C P^{1}$. This is the complex manifold obtained by patching together two copies $U, \tilde{U}$ of the complex plane $C$ with coordinates $\zeta, \widetilde{\zeta}$ related by $\widetilde{\zeta}=\zeta^{-1}$ on $U \cap \widetilde{U} \cong C \backslash\{0\}$. Relative to the coordinates $(a, b, c)$ of $S^{2}$ in $R^{3}$ the complex coordinate $\zeta$ is given by

$$
(a, b, c)=\left(\frac{1-\zeta \bar{\zeta}}{1+\zeta \bar{\zeta}}, \frac{\zeta+\bar{\zeta}}{1+\zeta \bar{\zeta}}, \frac{i(\zeta-\bar{\zeta})}{1+\zeta \bar{\zeta}}\right) .
$$

Define now the twistor space $Z$ of $M^{4 n}$ to be the product manifold $M^{4 n} \times S^{2}$ equipped with a complex structure $\underline{I}$ defined on the tangent space of $Z$ at $(m, \zeta)$ as follows: express the tangent space as a direct sum $T_{m} \oplus T_{\zeta}$ and define

$$
\mathbf{I}=\left(\frac{1-\zeta \bar{\zeta}}{1+\zeta \bar{\zeta}} \mathbf{I}+\frac{\zeta+\bar{\zeta}}{1+\zeta \bar{\zeta}} \mathbf{J}+i \frac{\zeta-\bar{\zeta}}{1+\zeta \bar{\zeta}} \mathbf{K}, I_{0}\right)
$$

where $I_{0}$ is the operation of multiplication by $i$ on the tangent space $T_{\zeta}$ of $\zeta \in S^{2}$.

To show that $Z$ actually admits complex coordinates, and is thus a complex manifold, we need the Newlander-Nirenberg theorem [17].

This theorem says that complex coordinates exist if for each $(1,0)$ form (i.e., complex 1 -form $\theta$ such that $\underline{I} \theta=i \theta$ ), the exterior derivative may be written in the form

$$
d \theta=\theta_{i} \wedge \alpha_{i}
$$

for $(1,0)$ forms $\theta_{i}$ and general 1 -forms $\alpha_{i}$. This is a complex version of the Frobenius integrability condition.

What, then, are the $(1,0)$ forms for the complex structure $\underline{I}$ ? Let $\varphi$ be a $(1,0)$ form for $\mathbf{I}$ on $M$, so that $\mathbf{I} \varphi=i \varphi$, and set

$$
\theta=\varphi+\zeta \mathbf{K} \varphi .
$$


Then,

$$
\begin{aligned}
(1+\zeta \bar{\zeta}) \underline{\mathbf{I}} \theta= & ((1-\overline{\zeta \zeta} \mathbf{I}+(\zeta+\bar{\zeta}) \mathbf{J}+i(\zeta-\bar{\zeta}) \mathbf{K}) \theta=(1-\zeta \bar{\zeta}) \mathbf{I} \varphi-\zeta(1-\zeta \bar{\zeta}) \mathbf{K} \mathbf{I} \varphi \\
& +(\zeta+\bar{\zeta}) \mathbf{K} \mathbf{I} \varphi+\zeta(\zeta+\bar{\zeta}) \mathbf{I} \varphi+i(\zeta-\bar{\zeta}) \mathbf{K} \varphi-i \zeta(\zeta-\bar{\zeta}) \varphi \\
= & i(1+\zeta \bar{\zeta})(\varphi+\zeta \mathbf{K} \varphi)
\end{aligned}
$$

where we have used $I \varphi=i \varphi$. Thus if $\varphi_{1}, \ldots, \varphi_{2 n}$ is a local basis of $(1,0)$ forms for $\mathbf{I}$, then $\varphi_{i}+\zeta \mathbf{K} \varphi_{i}(1 \leqq i \leqq 2 n)$ and $d \zeta$ give a basis for the $(1,0)$ forms of $Z$. Now consider

$$
d \theta=d(\varphi+\zeta \mathbf{K} \varphi)=d x^{i} \wedge \nabla_{\partial / \partial x^{i}}(\varphi+\zeta \mathbf{K} \varphi)+d \zeta \wedge \mathbf{K} \varphi .
$$

Since $\underline{I}$ is covariantly constant with respect to $\nabla_{\partial / \partial x^{i}}$,

$$
\underline{\mathbf{I}} \nabla_{\partial / \partial x^{i}}(\varphi+\zeta \mathbf{K} \varphi)=i \nabla_{\partial / \partial x^{i}}(\varphi+\zeta \mathbf{K} \varphi)
$$

so the first term in (3.75) is of the form $d x^{i} \wedge \theta_{i}$ for $(1,0)$ forms $\theta_{i}$. The second term is the product of the $(1,0)$ form $d \zeta$ and another 1 -form, so the whole expression is of the form (3.72) and the complex structure is integrable. Hence, by the NewlanderNirenberg theorem, the twistor space $Z$ has the structure of a complex manifold of dimension $(2 n+1)$. The projection

$$
p: Z \rightarrow C P^{1}
$$

is holomorphic and each copy $\left(m, C P^{1}\right)$ of the projective line is a holomorphic section of this projection. We call the sections twistor lines.

From the purely differential geometric point of view, the twistor space is still the product $M \times S^{2}$, and so the normal bundle to each twistor line (the vector bundle obtained as the quotient of the tangent vectors to $Z$ over the line by the tangent vectors to the line) is simply the trivial product bundle $S^{2} \times T_{m}$, where $T_{m}$ is the $4 n$-dimensional tangent space at $m \in M$. As a holomorphic vector bundle it is not trivial, however, and it is important in inverting the construction above to know what it is.

We take the $4 n$-dimensional vector space $T_{m}$ and represent the action of $\mathbf{I}, \mathbf{J}, \mathbf{K}$ on $T_{m} \cong R^{4 n} \cong C^{2 n}$ by the complex matrices

$$
\left(\begin{array}{cc}
i 1_{n} & 0 \\
0 & -i 1_{n}
\end{array}\right) \quad\left(\begin{array}{cc}
0 & 1_{n} \\
-1_{n} & 0
\end{array}\right) \quad\left(\begin{array}{cc}
0 & i 1_{n} \\
i 1_{n} & 0
\end{array}\right)
$$

and so

$$
\underline{\mathbf{I}}=\frac{1}{1+\zeta \bar{\zeta}}\left(\begin{array}{cc}
i(1-\zeta \bar{\zeta}) & 2 \zeta \\
-2 \zeta & -i(1-\zeta \bar{\zeta})
\end{array}\right)
$$

This matrix describes the complex structure on $T_{m}$ at a point $\zeta \in S^{2}$, so that the complex vectors over $\zeta$ consist of the eigenspace of $\underline{I}$ acting on $R^{4 n} \otimes C$ corresponding to the eigenvalue $i$, and these are vectors of the form:

$$
\left(\begin{array}{c}
v \\
i \zeta v
\end{array}\right)
$$


In fact, (3.73) tells us this: $\left(\begin{array}{c}v \\ i \zeta v\end{array}\right)=(1+\zeta \mathbf{K})\left(\begin{array}{l}v \\ 0\end{array}\right)$. Rewriting (3.79) in terms of the coordinate $\tilde{\zeta}=\zeta^{-1}$, the complex vectors may be written as

$$
\left(\begin{array}{c}
-i \widetilde{\zeta} w \\
w
\end{array}\right)
$$

so the vector bundle is obtained by taking $U \times C^{2 n}$ and $\tilde{U} \times C^{2 n}$ and patching together over $U \cap \widetilde{U}$ with the transition matrix $i \zeta \cdot 1_{2 n}$ or equivalently by change of basis $\zeta \cdot 1$.

Denote by $O(k)$ the holomorphic line bundle over $C P^{1}$ with transition function $\zeta^{k}$, then what we have found is that the normal bundle of each twistor line is holomorphically equivalent to $C^{2 n} \otimes O(1)$ [which we write as $C^{2 n}(1)$ ]. Since we have a holomorphic projection $p: Z \rightarrow C P^{1}$ we may pull back the bundle $O(k)$ from $C P^{1}$ to $Z$. We shall still denote it by the same symbol.

We are here collecting the essential holomorphic properties of the twistor space. The next one concerns the interpretation of the covariant constant 2-forms $\omega_{1}, \omega_{2}, \omega_{3}$. We saw in Sect. 3D that $\omega_{+}=\omega_{2}+i \omega_{3}$ was a holomorphic 2-form of type $(2,0)$ with respect to the complex structure $\mathbf{I}$. We may locally find a basis $\left\{\varphi_{i}\right\}$ of $(1,0)$ forms for I such that

$$
\frac{1}{2} \omega_{+}=\sum_{i=1}^{n} \varphi_{i} \wedge \varphi_{n+i}
$$

Consider now the complex 2-form (suppressing the summation symbol)

$$
\frac{1}{2} \omega=\left(\varphi_{i}+\zeta \mathbf{K} \varphi_{i}\right) \wedge\left(\varphi_{n+i}+\zeta \mathbf{K} \varphi_{n+i}\right) .
$$

From (3.73), $\omega$ is a 2 -form of type $(2,0)$ for the complex structure $\mathbf{I}(\zeta)$. It is quadratic in $\zeta$ :

$$
\frac{1}{2} \omega=\varphi_{i} \wedge \varphi_{n+i}+\zeta\left(\mathbf{K} \varphi_{i} \wedge \varphi_{n+i}+\varphi_{i} \wedge \mathbf{K} \varphi_{n+i}\right)+\zeta^{2} \mathbf{K} \varphi_{i} \wedge \mathbf{K} \varphi_{n+i}
$$

Now, evaluating on vectors $X, Y$,

$$
\begin{aligned}
2( & \left.\mathbf{K} \varphi_{i} \wedge \varphi_{n+i}+\varphi_{i} \wedge \mathbf{K} \varphi_{n+i}\right)(X, Y)=\varphi_{i}(\mathbf{K} X) \varphi_{n+i}(Y)-\varphi_{i}(\mathbf{K} Y) \varphi_{n+i}(X) \\
& +\varphi_{i}(X) \varphi_{n+i}(\mathbf{K} Y)-\varphi_{i}(Y) \varphi_{n+i}(\mathbf{K} X) \\
= & \omega_{+}(\mathbf{K} X, Y)+\omega_{+}(X, \mathbf{K} Y) \\
= & \left(\omega_{2}+i \omega_{3}\right)(\mathbf{K} X, Y)+\left(\omega_{2}+i \omega_{3}\right)(X, \mathbf{K} Y) \\
= & g(\mathbf{J K} X, Y)+i g\left(\mathbf{K}^{2} X, Y\right)+g(\mathbf{J} X, \mathbf{K} Y)+i g(\mathbf{K} X, \mathbf{K} Y) \\
= & 2 g(\mathbf{I} X, Y)=2 \omega_{1}(X, Y),
\end{aligned}
$$

where the quaternionic relation on $\mathbf{I}, \mathbf{J}, \mathbf{K}$ has been used. Similarly,

$$
\begin{aligned}
2\left(\mathbf{K} \varphi_{i} \wedge \mathbf{K} \varphi_{n+i}\right)(X, Y) & =\varphi_{i}(\mathbf{K} X) \varphi_{n+i}(\mathbf{K} Y)-\varphi_{i}(\mathbf{K} Y) \varphi_{n+i}(\mathbf{K} X) \\
& =\left(\omega_{2}+i \omega_{3}\right)(\mathbf{K} X, \mathbf{K} Y)=g(\mathbf{J K} X, \mathbf{K} Y)+i g\left(\mathbf{K}^{2} X, \mathbf{K} Y\right) \\
& =-g(\mathbf{J} X, Y)+i g(\mathbf{K} X, Y)=-\left(\omega_{2}-i \omega_{3}\right)(X, Y) .
\end{aligned}
$$

Thus (3.83) may be written as

$$
\omega=\left(\omega_{2}+i \omega_{3}\right)+2 \zeta \omega_{1}-\zeta^{2}\left(\omega_{2}-i \omega_{3}\right) .
$$


For each $\zeta \in C P^{1}, \omega$, which defines a holomorphic (in fact covariantly constant) complex symplectic form on the fibers of the projection $p: Z \rightarrow C P^{1}$, depends holomorphically on $\zeta$ in a quadratic manner. In global terms, $\omega$ is a holomorphic section of the vector bundle $\Lambda^{2} T_{F}^{*}(2)$ over $Z$, where

$$
T_{F}=\operatorname{Ker} d p: T Z \rightarrow T C P^{1}
$$

is the tangent bundle along the fibers. The $O(2)$ twist is a consequence of the quadratic dependence of $\omega$ on $\zeta$.

The final piece of information is the map

$$
\tau: M \times S^{2} \rightarrow M \times S^{2},
$$

defined by the antipodal map on the $S^{2}$ factor

$$
\tau(m, \zeta)=\left(m,-\frac{1}{\bar{\zeta}}\right) .
$$

This takes the complex structure $\underline{I}$ to its conjugate $-\underline{I}$. We call this a real structure (it may be compared with the action of complex conjugation on a complex manifold given by an equation with real coefficients). All the holomorphic data we have given (and in particular the twistor lines) are compatible with $\tau$.

The idea of twistor theory is not only to extract holomorphic objects from differential equations, but also to encode the original equations (in this case for a hyperkähler metric) in holomorphic form. What we have here extracted in terms of holomorphic properties of the twistor space is in fact sufficient to reconstruct the hyperkähler metric. This is expressed by the following theorem:

Theorem 3.3. Let $Z^{2 n+1}$ be a complex manifold such that

(i) $Z$ is a holomorphic fiber bundle $p: Z \rightarrow C P^{1}$ over the projective line,

(ii) the bundle admits a family of holomorphic sections each with normal bundle isomorphic to $C^{2 n} \otimes O(1)$,

(iii) there exists a holomorphic section $\omega$ of $\Lambda^{2} T_{F}^{*}(2)$ defining a symplectic form on each fiber,

(iv) $Z$ has a real structure $\tau$ compatible with (i), (ii), and (iii) and inducing the antipodal map on $C P^{1}$.

Then the parameter space of real sections is a 4n-dimensional manifold with a natural hyperkähler metric for which $Z$ is the twistor space.

Proof. To prove the theorem we first show that the parameter space of real sections (we will call them twistor lines) is a smooth manifold of dimension $4 n$, secondly construct a metric on it and thirdly prove that the metric is hyperkählerian.

Let $M$ denote the parameter space of twistor lines and $P_{m}$ a line corresponding to $m \in M$. An infinitesimal deformation of the section $P_{m}$ of $p: Z \rightarrow C P^{1}$ can be thought of as a holomorphic vector field $X$ over $P_{m}$ pointing along the fibers (see Fig. 4). This is a holomorphic section of the normal bundle $N$ of $P_{m}$ in $Z$. This way of thinking is made precise by a theorem of Kodaira [18] which asserts that if the sheaf cohomology group $\mathrm{H}^{1}\left(C P^{1} ; N\right)$ vanishes then every holomorphic section of the normal bundle may be integrated to a deformation of the twistor line, which makes the parameter space of all holomorphic sections of $Z$ a complex manifold 


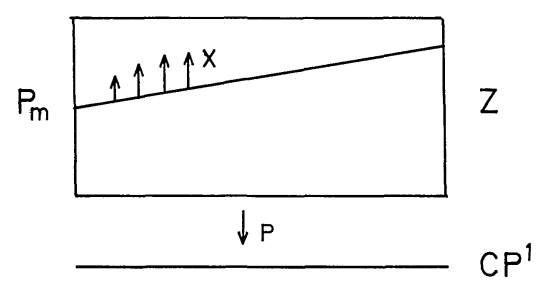

Fig. 4. The twistor space $Z$, viewed as a fibration over $C P^{1}$, and a deformation of a twistor line $P_{m}$ by a holomorphic vector field $X$, pointing along the fibers

with tangent space at a point $m$ isomorphic to the vector space of global holomorphic sections of $N$ along $P_{m}$. This space can also be written as $H^{0}\left(P_{m} ; N\right)$. In our case by assumption $N \cong C^{2 n} \otimes O(1)$ and the sheaf cohomology group $H^{1}\left(C P^{1} ; N\right) \cong C^{2 n} \otimes H^{1}\left(C P^{1} ; O(1)\right)$ does indeed vanish.

A global section of $O(1)$ on $C P^{1}$ is defined by holomorphic functions $\tilde{f}$ on $\tilde{U}$ and $f$ on $U$ such that (recall $\zeta=\widetilde{\zeta}^{-1}$ )

$$
f(\zeta)=\zeta \widetilde{f}\left(\frac{1}{\zeta}\right)
$$

on the region $U \cap \tilde{U}$, where $\zeta \neq 0$. Expanding $f$ and $\tilde{f}$ in power series, this forces $f$ to be linear and so every holomorphic section of $N \cong C^{2 n}(1)$ on $P_{m}$ is of the form

$$
s(\zeta)=a+b \zeta \quad\left(a, b \in C^{2 n}\right) .
$$

These sections form a $4 n$-dimensional complex vector space, so by the Kodaira theorem the twistor line $P_{m}$ lies in a $4 n$-dimensional family. The twistor lines which are real (i.e. preserved by $\tau$ ) are parametrized by a real $4 n$-dimensional submanifold $M$ whose tangent space at $m \in M$ is $T_{m}$, where

$$
T_{m} \otimes C=H^{0}\left(P_{m} ; N\right)=H^{0}\left(P_{m} ; T_{F}\right),
$$

recalling that $T_{F}$ is the tangent bundle along the fibers (or vertical bundle) of the fiber bundle $p: Z \rightarrow C P^{1}$.

We have thus constructed a manifold $M$ and identified its tangent space in terms of holomorphic data. Now we define the metric $g$ on $M$.

First note that by assumption, on $P_{m}$ we have

$$
T_{F} \cong C^{2 n} \otimes O(1),
$$

hence

$$
T_{F}(-1) \cong C^{2 n} \text {, }
$$

and we may rewrite (3.93) naturally as

$$
T_{m} \otimes C \cong H^{0}\left(P_{m} ; T_{F}(-1)\right) \otimes H^{0}\left(P_{m} ; O(1)\right) \cong C^{2 n} \otimes C^{2} .
$$

Now by hypothesis, the form $\omega \in H^{0}\left(Z ; \Lambda^{2} T_{F}^{*}(2)\right)$ defines a nondegenerate skew form on the $2 n$-dimensional space $H^{0}\left(P_{m} ; T_{F}(-1)\right)$ as we may regard it as a 2-form with values in $O(2)$. Also, the 2-dimensional space $H^{0}\left(P_{m} ; O(1)\right) \cong H^{0}\left(C P^{1} ; O(1)\right)$ has its own natural symplectic structure

$$
\left\langle a_{1}+b_{1} \zeta, a_{2}+b_{2} \zeta\right\rangle=a_{1} b_{2}-a_{2} b_{1} .
$$


We therefore define a complex inner product on $T_{m} \otimes C$ using these two symplectic forms:

$$
g(a+b \zeta, a+b \zeta)=2 \omega(a, b) .
$$

Formula (3.98) defines a complex inner product - we need to know the real tangent vectors, which will be tangential to the space of real lines.

A real structure on a complex vector space $V$ is an operation of complex conjugation. It is a real linear map $t: V \rightarrow V$ such that $t^{2}=1$ and $t(\lambda v)=\bar{\lambda} t(v)$. Closely related to this is the notion of quaternionic structure on $V$. This is a real linear map $j: V \rightarrow V$ such that

$$
j^{2}=-1, \quad j(\lambda v)=\bar{\lambda} j(v) .
$$

Since $j i=-i j$, the transformations $i, j, i j=k$ generate an operation of the quaternions on $V$.

From this point of view it is easy to see that the tensor product of two complex vector spaces with quaternionic structures is a vector space with a real structure. Since the tangent space $T_{m} \otimes C$ is expressed in (3.96) as the tensor product of two complex vector spaces, we may define a real structure on $T_{m} \otimes C$ by taking quaternionic structures on the spaces $H^{0}\left(P_{m} ; T_{F}(-1)\right)$ and $H^{0}\left(P_{m} ; O(1)\right)$. Consider first the 2-dimensional space $H^{0}\left(C P^{1} ; O(1)\right)$. The map $j$ defined by

$$
j(a+b \zeta)=\bar{b}-\bar{a} \zeta
$$

is a quaternionic structure. It is obtained from the unique (modulo \pm ) action of the holomorphic line bundle $O(1)$ which covers the action $\tau: C P^{1} \rightarrow C P^{1}$ given by $\tau(\zeta)=-1 / \bar{\zeta}$

The real structure $\tau$ preserves the tangent bundle along the fibers and the natural decomposition (3.96) and so induces a quaternionic structure $j$ on the $2 n$-dimensional complex vector space $H^{0}\left(P_{m} ; T_{F}(-1)\right)$. Thus, in the form (3.92) the real structure is

$$
t(a+b \zeta)=j b-j a \zeta,
$$

and so a real tangent vector can be written as

$$
X=a-j a \zeta, \quad a \in H^{0}\left(P_{m} ; T_{F}(-1)\right),
$$

and then from (3.98) the metric is given by

$$
g(X, X)=-2 \omega(a, j a) .
$$

The compatibility of $\omega$ with $\tau$ in the theorem is the statement that this is a positive definite metric.

Thus far we have obtained the $4 n$-dimensional manifold $M$ as an abstract parameter space of real twistor lines, and constructed a metric on $M$. In fact the foregoing arguments allow us a more explicit identification of $M$ : namely with an open set in any one of the fibers of the bundle $p: Z \rightarrow C P^{1}$.

We consider a real holomorphic section of the normal bundle of $P_{m}$. This is written as

$$
X=a-(j a) \zeta .
$$


Suppose $X$ vanishes at $\zeta=\zeta_{0}$, then from (3.103),

$$
g(X, X)=-2 \omega\left(\zeta_{0}(j a), j a\right)=-2 \zeta_{0} \omega(j a, j a)=0 .
$$

But $g$ is positive definite, so $X$ must vanish identically. Consequently infinitesimal deformations of real twistor lines do not vanish anywhere along the line. They therefore separate points in the fibers of $Z$, and neighbouring real lines intersect the fibers of $Z$ in distinct points. Thus the $4 n$-dimensional manifold $M$ of real lines can (locally) be identified with any one of the fibers of $Z$. We shall use this fact to help in proving the metric defined above is hyperkählerian, which is the final step in the proof.

First identify $M$ with the fiber $Z_{0}$ over $\zeta=0$ in $C P^{1}$. Then the real tangent vector $X=a-(j a) \zeta$ at $\zeta=0$ is identified with $a \in H^{0}\left(P_{m} ; T_{F}(-1)\right)$. Since $T_{F}(-1)$ $\cong C^{2 n}$, the trivial bundle, the section $a$ is determined by its value at any point $\zeta \in C P^{1}$ and in particular at $\zeta=0$. Here $T_{F}$ is just the tangent bundle of the fiber $Z_{0}$. The map $X \rightarrow a$ is the derivative of the identification of $M$ with $Z_{0}$.

The fiber $Z_{0}$ is a complex manifold and so defines a complex structure I on $M$ under the identification, corresponding to multiplying $a$ by $i$. Hence, considering the compatibility of $\mathbf{I}$ with the metric $g$, we have

$$
\begin{aligned}
g(\mathbf{I} X, Y) & =-\omega(i a, j b)-\omega(b, j i a)=-i \omega(a, j b)+i \omega(b, j a) \\
& =\omega(a, j i b)+\omega(i b, j a)=-g(X, \mathbf{I} Y) .
\end{aligned}
$$

Thus the metric $g$ is hermitian with respect to the integrable complex structure $\mathbf{I}$.

Consider next the complex symplectic 2 -form $\omega$ on the fiber over $\zeta=-1$. This defines a form on $M$

$$
\varphi_{-1}(X, Y)=\omega(a+j a, b+j b)
$$

and at $\zeta=+1$

$$
\varphi_{+1}(X, Y)=\omega(a-j a, b-j b)
$$

Thus

$$
\frac{1}{2}\left(\varphi_{-1}-\varphi_{+1}\right)(X, Y)=\omega(j a, b)+\omega(a, j b)=i g(\mathbf{I} X, Y) .
$$

Now both $\varphi_{1}$ and $\varphi_{-1}$ were closed, so the hermitian form $g(\mathbf{I} X, Y)$ is closed, and thus the metric $g$ is Kähler with respect to $\mathbf{I}$.

Repeating with $\mathbf{J}$ and $\mathbf{K}$ shows the metric to be hyperkählerian, which concludes the proof of Theorem 3.3.

This twistorial setting provides a natural context for the hyperkähler quotient construction of Sect. 3D. We may ask how the twistor spaces $Z$ and $\hat{Z}$ of a hyperkähler manifold $M$ and its quotient $\hat{M}$ by a group $G$ are related. If $G$ is a group of isometries of $M$ which preserve $\mathbf{I}, \mathbf{J}$, and $\mathbf{K}$, then the vector fields generated by $G$ are actually holomorphic on $Z=M \times S^{2}$ with the complex structure $\underline{\mathbf{I}}$. If we suppose that this Lie algebra action extends to an action of the complexified group $G^{C}$, then the quotient construction produces the twistor space $\hat{Z}$ from $Z$ by taking the holomorphic symplectic quotient along the fibers of $Z$, with respect to the holomorphic symplectic form $\omega$. Note that since $\omega$ is a section of $\Lambda^{2} T_{F}^{*}(2)$ and is a symplectic form twisted by $O(2)$, the holomorphic moment map for the action of $G^{C}$ is a holomorphic section of $\mathrm{g}^{*} \otimes O(2)$ over $Z$. 


\section{(G) The Legendre Transform}

We shall next use the twistor space approach to derive the Legendre transform construction of hyperkähler metrics. We shall see how the contour integral expression (2.2) arises naturally in this framework. The holomorphic function which appears in the contour integral arises in the twistor theory approach as the Hamiltonian function $H$ for a symplectic vector field which we exponentiate to obtain a symplectic diffeomorphism. This diffeomorphism patches together two copies of $C^{2 n+1}$ to get a non-trivial twistor space. The function $H$ depends holomorphically on $n+1$ variables, and in some sense the most general twistor space for a hyperkähler manifold is obtained by extending the construction to a holomorphic function of all $2 n+1$ variables. The explicit form of the Kähler potential is, however, more readily obtained in the restricted situation which follows. What the Legendre transform constructs is the general hyperkähler metric in dimension $4 n$ with $n$ commuting Killing fields which preserve $\mathbf{I}, \mathbf{J}$, and $\mathbf{K}$.

We begin then with a hyperkähler manifold $M^{4 n}$ with a free action of the additive Lie group $R^{n}$ on it, preserving the hyperkähler structure. Let $Z$ be the twistor space of $M$ and assume that the holomorphic action of $R^{n}$ extends to a free action of $C^{n}$. As discussed above, we then obtain moment maps $\mu_{1}, \ldots, \mu_{n}$ which are holomorphic sections of $O(2)$ on $Z$.

Since the group is abelian, each $\mu_{i}$ is invariant under the group and they define a holomorphic map

$$
\mu: Z \rightarrow Y,
$$

where $Y=C^{n} \otimes O(2)$ is the total space of the vector bundle over $C P^{1}$ with transition matrix $\zeta^{2} \cdot 1_{n}$. The map $\mu$ is invariant under the action of $C^{n}$, and each $n$-dimensional fiber is an orbit of the group. In other words, assuming the map $\mu$ is surjective, we have represented $Z$ as a principal bundle over $Y$ with structure group the additive group $C^{n}$.

Now $Y$ is obtained by patching together $C^{n} \times U$ and $C^{n} \times \widetilde{U}$ with transition function $\zeta^{2} \cdot 1_{n}$, so we have holomorphic coordinates $\left(\eta^{i}, \zeta\right)$ on $C^{n} \times U$ and $\left(\tilde{\eta}^{i}, \widetilde{\zeta}\right)$ on $C^{n} \times \tilde{U}$ related by

$$
\tilde{\eta}^{i}=\zeta^{-2} \eta^{i}, \quad \tilde{\zeta}=\zeta^{-1}
$$

on $C^{n} \times U \cap \tilde{U}$. In these coordinates, the projection $\mu$ is just $\eta$.

The principal bundle $Z$ over $Y$ can equally be given in terms of transition functions. We have coordinates $\left(\xi^{i}, \eta^{i}, \zeta\right)$ on $C^{2 n} \times U$ and $\left(\tilde{\xi}^{i}, \tilde{\eta}^{i}, \widetilde{\zeta}\right)$ on $C^{2 n} \times \tilde{U}$ related by

$$
\xi^{i}=\xi^{i}+f^{i}\left(\eta^{j}, \zeta\right), \quad \tilde{\eta}^{i}=\zeta^{-2} \eta^{i}, \quad \tilde{\zeta}=\zeta^{-1}
$$

on $C^{2 n} \times U \cap \tilde{U}$. The functions $f^{i}$ represent the group action (translation) for the abelian group $C^{n}$. Looking again at the group action it is clear that the vector fields generated by it are, in these coordinates, the fields $\partial / \partial \xi^{i}$. The moment map $\mu_{i}$ in these coordinates is $\eta^{i}$, and hence the symplectic form along the fibers is given by

$$
\omega=2 d \xi^{i} \wedge d \eta^{i}=2 \zeta^{2} d \tilde{\xi}^{i} \wedge d \tilde{\eta}^{i} \quad(\text { modulo } d \zeta),
$$

where the last equality follows because $\omega$ is $O(2)$-valued. Hence, from (3.112)

$$
\frac{\partial f^{i}}{\partial \eta^{j}} d \eta^{j} \wedge d \eta^{i}=0,
$$


and we may write

$$
f^{i}=\frac{\partial H}{\partial \eta^{i}} .
$$

Note that the symplectic transformation (3.112) which patches together the two copies of $C^{2 n+1}$ is obtained by exponentiating the symplectic vector field

$$
\frac{\partial H}{\partial \eta^{i}} \frac{\partial}{\partial \xi^{i}}
$$

corresponding to the Hamiltonian $H$.

To calculate the hyperkähler metric according to the procedure of Theorem 3.3 . we need the real structure. Modulo a sign (or a choice of new coordinates) this is determined by the antipodal map $\zeta \rightarrow-1 / \bar{\zeta}$ and the transition relation (3.112). We obtain

$$
\tau(\zeta)=-\frac{1}{\bar{\zeta}}, \quad \tau\left(\eta^{i}\right)=-\frac{\bar{\eta}^{i}}{\bar{\zeta}^{2}}, \quad \tau\left(\xi^{i}\right)=-\overline{\xi^{i}} .
$$

We now find the twistor lines. Each one when projected by $\mu$ to $Y=C^{n} \otimes O(2)$ becomes a holomorphic section of this vector bundle. A holomorphic section of $O(2)$ over $C P^{1}$ is given by functions $\widetilde{f}$ on $\widetilde{U}$ and $f$ on $U$ such that

$$
\zeta^{2} \tilde{f}\left(\frac{1}{\zeta}\right)=f(\zeta) \quad \text { when } \zeta \neq 0
$$

Again (as in Theorem 3.3) comparing coefficients in the power series of each, the only global sections are quadratic polynomials:

$$
f(\zeta)=a+b \zeta+c \zeta^{2} .
$$

Consequently, the twistor lines satisfy

$$
\eta^{i}=a^{i}+b^{i \zeta}+c^{i \zeta^{2}}
$$

and the real ones from (3.117) can be written

$$
\eta^{i}=z^{i}-x^{i \zeta}-\bar{z}^{i} \zeta^{2}
$$

for $x^{i} \in R, z^{i} \in C$. The function $\left(x^{i}, z^{i}\right) \rightarrow R^{3} \otimes R^{n}$ is actually the moment map for the group $R^{n}$ acting on $M$.

We know now the projections of the twistor lines to $Y$. They are given by (3.121) and depend on the $3 n$ parameters $\left(x^{i}, z^{i}\right) \in R^{n} \times C^{n}$. To find the full $4 n$-parameter family we require a 1-parameter family of lines in $Z$ which project to a fixed line in $Y$. From the description of the twistor space $Z$ in terms of the patching function (3.112) this requires finding a holomorphic function $\tilde{\xi}^{i}$ of $\tilde{\zeta}$ and a function $\xi^{i}$ of $\zeta$ which satisfy

$$
\xi^{i}\left(\frac{1}{\zeta}\right)=\xi^{i}(\zeta)+\frac{\partial H}{\partial \eta^{i}}\left(\eta^{1}(\zeta), \ldots, \eta^{n}(\zeta), \zeta\right),
$$

where $\eta^{i}(\zeta)=z^{i}-x^{i} \zeta-\bar{z}^{i} \zeta^{2}$ for fixed $x^{i}, z^{i}$. Expanding in power series,

$$
\xi^{i}=\sum_{n=0}^{\infty} a_{n}^{i \zeta^{-n}} \text { and } \xi^{i}=\sum_{n=0}^{\infty} b_{n}^{i} \zeta^{n}
$$


where the coefficients are determined by considering the Laurent expansion of

$$
\frac{\partial H}{\partial \eta^{i}}\left(\eta^{1}, \ldots, \eta^{n}, \zeta\right)
$$

in positive and negative powers of $\zeta$ and assigning to $\tilde{\xi}^{i}$ the negative powers and to $\xi^{i}$ the positive powers. The only coefficients that are not uniquely determined are the $\zeta^{0}$-coefficients $a_{0}^{i}$ and $b_{0}^{i}$. The 1-dimensional ambiguity in choice (for each $i$ ) gives the extra degree of freedom to obtain a $4 n$-dimensional parameter space. The reality condition (3.117) forces this choice to be one real dimension: more explicitly, reality gives

$$
a_{0}^{i}=-\overline{b_{0}^{i}} .
$$

Thus a constant $c$ can be added to both sides of (3.122) to preserve reality only if

$$
c=-\bar{c} .
$$

Equation (3.122) gives the following relation between $a_{0}^{i}$ and $b_{0}^{i}$ :

$$
a_{0}^{i}-b_{0}^{i}=\frac{1}{2 \pi i} \int_{C} \frac{\partial H}{\partial \eta^{i}} \frac{d \zeta}{\zeta}
$$

where $C$ is a contour separating $\zeta=0$ and $\zeta=\infty$. We shall use this expression to find the metric using the prescription of Theorem 3.3 in terms of the complex structure at $\zeta=0$.

The real twistor lines intersect the fiber of $Z \rightarrow C P^{1}$ over $\zeta=0$ at a point with coordinates [cf. (3.121)]

$$
\eta^{i}(0)=z^{i}, \quad \xi^{i}(0)=u^{i}
$$

which we know by construction are holomorphic with respect to the complex structure $\mathbf{I}=\underline{\mathbf{I}}(\zeta=0)$. The function $x^{i}$ in (3.121) is now defined as a function of $z^{j}$ and $u^{j}$ which we need to determine. This is achieved by (3.127). If we set

$$
F\left(x^{i}, z^{i}, \bar{z}^{i}\right)=\frac{1}{2 \pi i} \int_{C} H\left(\eta^{1}, \ldots, \eta^{n}, \zeta\right) \frac{d \zeta}{\zeta^{2}},
$$

and put $\eta^{i}=z^{i}-x^{i} \zeta-\bar{z}^{i} \zeta^{2}$ in this expression, then from (3.127)

$$
\frac{\partial F}{\partial x^{i}}=\frac{1}{2 \pi i} \int_{C} \frac{\partial H}{\partial \eta^{i}}(-\zeta) \frac{d \zeta}{\zeta^{2}}=-\left(a_{0}^{i}-b_{0}^{i}\right) .
$$

But

$$
u^{i}=\xi^{i}(0)=b_{0}^{i}
$$

and from (3.125)

$$
a_{0}^{i}=-\bar{u}^{i}
$$

hence

$$
\frac{\partial F}{\partial x^{i}}=u^{i}+\bar{u}^{i}
$$

This is the equation which determines $x^{i}$ as a function of $u^{i}$ and $z^{i}$. 
To find the Kähler form corresponding to I we take the holomorphic form

$$
\frac{1}{2} \omega=d \xi^{i} \wedge d \eta^{i}
$$

along the fibers of $Z$. From (3.117) and (3.113) the reality condition

$$
\tau^{*} \omega=\bar{\omega} \bar{\zeta}^{-2}
$$

where $\tau^{*}$ is the usual pull-back operation on forms along the fibers. For

$$
\varphi=\left(\omega_{2}+i \omega_{3}\right)+2 \omega_{1} \zeta-\left(\omega_{2}-i \omega_{3}\right) \zeta^{2},
$$

we find

$$
\tau^{*} \varphi=\left(\omega_{2}+i \omega_{3}\right)-2 \omega_{1} \bar{\zeta}^{-1}-\left(\omega_{2}-i \omega_{3}\right) \bar{\zeta}^{-2}=-\bar{\varphi} \bar{\zeta}^{-2} .
$$

Hence, up to a real constant, we have the Kähler form $\omega_{1}$ determined by the coefficient of $\zeta$ in

$$
\begin{aligned}
\frac{i}{2} \omega & =i d \xi^{i} \wedge d \eta^{i}=i\left(d u^{i}+d b_{1}^{i} \zeta+\ldots\right) \wedge\left(d z^{i}-d x^{i \zeta}+\ldots\right) \\
& =i d u^{i} \wedge d z^{i}+i \zeta\left(d b_{1}^{i} \wedge d z^{i}-d u^{i} \wedge d x^{i}\right)+\ldots
\end{aligned}
$$

Now $b_{1}^{i}$ is the coefficient of $\zeta$ in $\xi^{i}(\zeta)$ and this, by the definition of $\xi^{i}(\zeta)$ above is the negative of the corresponding coefficient in $\partial H / \partial \eta^{i}$. Thus, from (3.129)

$$
b_{1}^{i}=-\frac{1}{2 \pi i} \int_{C} \frac{\partial H}{\partial \eta^{i}} \frac{d \zeta}{\zeta^{2}}=-\frac{\partial F}{\partial z^{i}} .
$$

Thus from (3.134) and (3.138) the Kähler form $\omega_{1}$ is given by

$$
\omega_{1}=-2 i\left(d\left(\frac{\partial F}{\partial z^{i}}\right) \wedge d z^{i}+d u^{i} \wedge d x^{i}\right) .
$$

But if

$$
K=F-x^{i} \frac{\partial F}{\partial x^{i}}
$$

then

$$
\partial K=\frac{\partial F}{\partial z^{i}} d z^{i}+\frac{\partial F}{\partial x^{i}} \partial x^{i}-\partial x^{i} \frac{\partial F}{\partial x^{i}}-x^{i} \partial\left(\frac{\partial F}{\partial x^{i}}\right)=\frac{\partial F}{\partial z^{i}} d z^{i}-x^{i} \partial\left(\frac{\partial F}{\partial x^{i}}\right)
$$

and

$$
\begin{aligned}
\bar{\partial} \partial K & =\frac{\partial^{2} F}{\partial \bar{z}^{j} \partial z^{i}} d \bar{z}^{j} \wedge d z^{i}+\frac{\partial^{2} F}{\partial x^{j} \partial z^{i}} \bar{\partial} x^{j} \wedge d z^{i}-\bar{\partial} x^{i} \wedge \partial\left(\frac{\partial F}{\partial x^{i}}\right)-x^{i} \bar{\partial} \partial\left(\frac{\partial F}{\partial x^{i}}\right) \\
& =\bar{\partial}\left(\frac{\partial F}{\partial z^{i}}\right) \wedge d z^{i}+d u^{i} \wedge \bar{\partial} x^{i},
\end{aligned}
$$

using (3.133) to write $\partial F / \partial x^{i}=u^{i}+\bar{u}^{i}$. Thus, knowing that (3.140) is of type $(1,1)$, this gives $K$ as the Kähler potential. 
Remark. The above geometrical description has made some regularity assumptions [e.g., the existence of a free $C^{n}$-action and the description of the corresponding principal bundle over the whole of $\left.Y=C^{n} \otimes O(2)\right]$. This is an aspect of twistor theory which is useful in making particular boundary conditions correspond to different regularity conditions on the holomorphic objects which describe them. In practice, however, some of the more interesting hyperkähler metrics constructed by the Legendre transform do not satisfy these conditions. This means that we must analyze what we have done here purely in terms of differential equations, and note that the equation

$$
K=F-x^{i} \frac{\partial F}{\partial x^{i}}
$$

for a function $F\left(x^{i}, z^{i}, \bar{z}^{i}\right)$ defined by a contour integral

$$
F=\frac{1}{2 \pi i} \int_{C} H\left(\eta^{1}, \ldots, \eta^{n}, \zeta\right) \frac{d \zeta}{\zeta^{2}}
$$

does indeed provide the Kähler potential for a hyperkähler metric, even if $H$ is not a single-valued holomorphic function defined on the whole of $C^{n} \times C \backslash\{0\}$. This allows the more complicated integrals described in Sect. 2A.

\section{Nonlinear $\sigma$-Models}

In this section we introduce the basics of nonlinear $\sigma$-models and discuss duality and gauging of isometries. The constructions of hyperkähler manifolds follow directly from the supersymmetric generalization of these concepts.

\section{(A) Basics}

Fields $\varphi$ in a field theory are sections of a bundle $E$ with fiber $F$ over a base space $M$. We will call the fiber $F$ the target space for the maps $\varphi$. In familiar examples $M$ is the space-time manifold, and, for scalar field theory $F$ is a linear space, for spinor field theory $E$ is a spin bundle, and for Yang-Mills theory with gauge group $G, E$ is an affine bundle whose sections are connections on a principal $G$-bundle over $M$. In general, the base space has a metric and the target space is also assumed to have some geometrical properties. In our case, the base space will be either flat Minkowski or Euclidean space or superspace (see next section), while the target space will be a Riemannian space or a direct product of a Riemannian space and some other space.

The dynamics of the fields $\varphi$ are determined by an action functional $S$

$$
S=\int_{M} L(\varphi(x)), \quad x \in M, \quad \varphi(x) \in F .
$$

The Lagrangian density $L$ is a function of $\varphi(x)$ and its derivatives. For the scalar field theories we consider, the action is (here the bundle $E$ is trivial, i.e., a direct product $M \times F)$ [19]

$$
S=-\frac{1}{2} \int d^{D} x g_{i j}(\varphi) \partial^{a} \varphi^{i} \partial_{a} \varphi^{j}, \quad \partial_{a} \equiv \frac{\partial}{\partial x^{a}}, \quad a=1, \ldots, D,
$$


where $M$ is $D$-dimensional Euclidean or Minkowski space with (Cartesian) coordinates $x^{a}$ and $g_{i j}$ is the metric on the target space $F$ with coordinates $\varphi^{i}$.

For historical reasons, theories such as (4.2) are called nonlinear $\sigma$-models. $F$ is often taken to be a coset space. If $g$ is the flat metric, then the theory is free.

Field equations follow from extremizing the action functional with respect to $\varphi$. For the action (4.2):

$$
\partial^{a} \partial_{a} \varphi^{i}+\Gamma_{j k}^{i} \partial^{a} \varphi^{j} \partial_{a} \varphi^{k}=0
$$

where $\Gamma_{j k}^{i}$ is the Levi-Civita (Christoffel) connection on $F$. Mathematicians call $\varphi$ 's that satisfy (4.3) harmonic maps because they satisfy a generalized Laplace equation, and physicists think of them as being on-shell ${ }^{2}$ fields because they satisfy a field equation. For a free theory, in suitable coordinates $\varphi^{i}$ on $F$, the field equations (4.3) are linear and the general solution is a superposition of plane waves.

In general, one considers action functionals that are invariant (modulo boundary terms) under some symmetries. Continuous symmetries are most often given as infinitesimal transformations of the fields. For example, translations in a flat space $M$ can be written as

$$
\delta \varphi=-\xi^{a} \partial_{a} \varphi,
$$

where $\xi^{a}$ is a constant parameter of the transformation. Under this transformation the Lagrangian varies as a total derivative:

$$
\delta S=-\int_{M} \xi^{a} \partial_{a} L
$$

Such terms are usually thrown away by assuming that derivatives of fields fall off sufficiently fast at infinity. This is a space-time symmetry, i.e., a symmetry of the base space $M$. An internal symmetry acts on $\varphi$ at each point of $M$, i.e., it acts without space-time derivative. For example, a $U(1)$-algebra acts on a complex field $\varphi$ as:

$$
\delta \varphi=i \lambda \varphi
$$

If the transformation parameters, e.g., $\xi^{a}$ and $\lambda$, are constants over $M$ (and hence of course, over $F$ ) then the transformations are called global or rigid. This use of these words must not be confused with their mathematical usage. The group of transformations is called local if the parameters are arbitrary functions over $M$, $\lambda=\lambda(x)$ (e.g., gauge transformations of Yang-Mills fields). Sometimes, it is possible to modify a theory and promote a global symmetry to a local one; the prescription for doing this is called gauging the symmetry, and is discussed extensively below.

Given two or more infinitesimal symmetry transformations of $\varphi$, the commutator $\left[\delta_{1}, \delta_{2}\right] \varphi=\delta_{1}\left(\delta_{2} \varphi\right)-\delta_{2}\left(\delta_{1} \varphi\right)$ generates another symmetry. The algebra of symmetry transformations may be finite or infinite dimensional. In many cases of interest it is finite dimensional, e.g. (4.4) and (4.6) above. Even if it is infinite dimensional when acting on arbitrary fields $\varphi$, it may happen that it acts as a finite

\footnotetext{
${ }^{2}$ Note that, in scattering theory, on-shell is used for a field satisfying a free field equation even when the theory is nonlinear
} 
dimensional algebra on fields that are solutions to the field equations (4.3), i.e., on harmonic maps. In this case the transformations are said to form an on-shell realization of the finite algebra. The distinction between on-shell and off-shell realizations of an algebra is important in supersymmetry [20]. Whereas for onshell realizations the transformations $\delta \varphi$ depend on the detailed form of the action functional, for off-shell realizations the transformation laws are general, and typically leave families of actions invariant.

We now specialize the discussion of symmetries to the nonlinear $\sigma$-models in (4.2). The space-time symmetries of these models are the isometries of the flat $D$-dimensional Euclidean or Minkowski space $M$, i.e., the Poincaré group. The internal symmetries are the isometries of the target space $F$. These are global symmetries generated by the Killing vectors of $F$ :

$$
\delta \varphi^{i}=X^{A} k_{A} \varphi^{i}=X^{A} k_{A}^{i},
$$

where $X^{A}$ are constant parameters and $k_{A} \equiv k_{A}^{i} \partial_{i}$ satisfy Killing's Eq. (1.5) and generate the Lie algebra of the isometry group (1.7).

\section{(B) Duality}

We now discuss duality transformations, for refs. see $[21,22]$. The Legendre transform construction of hyperkähler metrics of Sect. 6 is the naive supersymmetric extension of the procedure described in this subsection. If the action (4.2) has a continuous internal symmetry, i.e., the target space $F$ has a Killing vector field, then we can perform a duality transformation of the action. This replaces a scalar field by a gauge ( $D-2)$-form and interchanges the role of the field equations and Bianchi identities. As this affects the field equations, it may change an off-shell realization into an on-shell one, or vice versa.

When there is a Killing vector field, we can choose (local) coordinates on $F$ such that the Killing vector is $k=\partial / \partial \varphi^{0}$. The components of the metric $g$ are then independent of $\varphi^{0}$, and hence the action depends on $\varphi^{0}$ only through $\partial_{a} \varphi^{0}$. We can then rewrite the action in first order ${ }^{3}$ form by substituting for $\partial_{a} \varphi^{0}$ a new field $V_{a}$ and introducing a Lagrange multiplier $T^{a b}$ :

$$
S=-\frac{1}{2} \int d^{D} x\left(g_{00} V^{a} V_{a}+2 g_{0 i} V^{a} \partial_{a} \varphi^{i}+g_{i j} \partial^{a} \varphi^{i} \partial_{a} \varphi^{j}+T^{a b} \partial_{a} V_{b}\right),
$$

where $i, j \neq 0$ and $T^{a b}=-T^{b a}$. Extremizing (4.8) with respect to $T^{a b}$ gives $\partial_{[a} V_{b]}=0$ and hence, locally, $V_{a}=\partial_{a} \varphi^{0}$. Substituting this solution into (4.8) gives (4.2) back. Extremizing (4.8) with respect to $V_{a}$ we find $V_{a}$ as a function of $T^{a b}$ and $\varphi^{i}$ :

$$
V_{a}=-\left(g_{00}\right)^{-1}\left(\frac{1}{2} \partial_{b} T_{a}^{b}+g_{0 i} \partial_{a} \varphi^{i}\right) .
$$

Substituting (4.9) into the first order action (4.8) gives (modulo boundary terms)

$$
\begin{aligned}
S= & -\frac{1}{2} \int d^{D} x\left[\left(g_{00}\right)^{-1}\left(-\frac{1}{4} \partial^{b} T_{b}^{a} \partial^{c} T_{c a}+g_{0 i} \partial^{a} \varphi^{i} \partial^{b} T_{b a}\right)\right. \\
& \left.+\left(g_{i j}-\left(g_{00}\right)^{-1} g_{0 i} g_{0 j}\right) \partial^{a} \varphi^{i} \partial_{a} \varphi^{j}\right] .
\end{aligned}
$$

\footnotetext{
${ }^{3}$ It is called first order because the term $\left(\partial_{a} \varphi^{0}\right)^{2}$, which is second order in derivatives, has been replaced by terms at most first order in derivatives
} 
Observe that the procedure we have followed is precisely a Legendre transformation of the Lagrangian in (4.2) with respect to $\partial_{a} \varphi^{0}$. The scalar field $\varphi^{0}$ has now been completely removed from the action and replaced by the antisymmetric tensor $T^{a b}$, which enters (4.10) through $\partial_{b} T^{a b}$. This can be rewritten as a field strength, i.e., as the exterior derivative of a tensor, by introducing the Hodge dual *T:

$$
\begin{aligned}
{ }^{*} T_{c_{1} \ldots c_{D-2}} & \equiv \frac{1}{2} \varepsilon_{c_{1} \ldots c_{D-2} a b} T^{a b}, \\
\partial_{b} T^{a b} & =\frac{1}{(D-2) !} \varepsilon^{a b c_{1} \ldots c_{D-2}} \partial_{b}^{*} T_{c_{1} \ldots c_{D-2}} .
\end{aligned}
$$

${ }^{*} T$ is a $(D-2)$-form, which, via the Legendre transformation, is said to be dual to the scalar field $\varphi^{0}$. The field equation that follows from extremizing the original action (4.2) with respect to $\varphi^{0}$ is

$$
\partial_{a}\left(g_{00} V^{a}\left(\varphi^{0}\right)+g_{0 i} \partial^{a} \varphi^{i}\right)=0, \quad V^{a}\left(\varphi^{0}\right) \equiv \partial^{a} \varphi^{0},
$$

whereas the field equation that follows from extremizing the dual action (4.10) with respect to $T^{a b}$ is

$$
\partial^{[a} V^{b]}(T)=0
$$

with $V^{a}(T)$ given by (4.9). For $V^{a}\left(\varphi^{0}\right)$ as in (4.12), (4.13) is a Bianchi identity, whereas for $V^{a}(T)$ as in (4.9), (4.12) is a Bianchi identity. Thus the duality transformation has interchanged the field equation and the Bianchi identity.

If the target space $F$ has several commuting Killing vector fields then each of the corresponding scalar fields can be exchanged for a $(D-2)$-form by a Legendre transformation.

\section{(C) Gauging}

We now discuss local internal symmetries and gauging. The quotient construction of hyperkähler metrics of Sect. 6 is the naive supersymmetric extension of the procedure described in this subsection. The basic idea is to promote a global symmetry of the action to a local symmetry by introducing a connection for the symmetry group. Explicitly, the action (4.2) is made invariant under the transformations (4.7) with $X^{A}=X^{A}(x)$ by replacing ordinary derivatives with covariant derivatives

$$
\partial_{a} \varphi^{i} \rightarrow \nabla_{a} \varphi^{i} \equiv \partial_{a} \varphi^{i}+A_{a}^{B} k_{B}^{i}
$$

where $A_{a}^{B}$ transforms as

$$
\delta A_{a}^{B}=-\partial_{a} X^{B}(x)+c_{C D}{ }^{B} A_{a}^{C} X^{D}(x)
$$

which guarantees the invariance of the action. The fields $A_{a}^{B}$ are gauge connections or Yang-Mills potentials and are components of Lie-algebra valued 1-forms. The substitution (4.14) is known as minimal coupling. The space-time dependent gauge transformation parameters $X^{A}(x)$ can be used to eliminate some scalar fields $\varphi^{i}$ from the action [cf. (4.7)]. The remaining scalar fields parametrize the space of $G$-orbits on $F$. We do not include a kinetic term $\frac{1}{4} F_{a b} F^{a b}$, where $F_{a b}$ is the curvature (field strength) of the connection $A$. 
We can reduce the gauged model to a nonlinear $\sigma$-model with target space $F_{0}$ (the space of $G$-orbits on $F$ ) by choosing a special connection that is a function of the scalar fields and substituting this connection back into the action. This connection is obtained by extremizing the gauged action with respect to $A_{a}^{B}$ :

$$
\begin{aligned}
A_{a}^{B} & =A_{i}^{B} \partial_{a} \varphi^{i} \equiv-g_{i j} k_{C}^{j} H^{B C} \partial_{a} \varphi^{i}, \\
H^{B C} & =\left(g_{i j} k_{B}^{i} k_{C}^{j}\right)^{-1} .
\end{aligned}
$$

The connection $A_{a}^{B}=\varphi^{*}\left(A_{i}^{B}\right)$ is the pullback to spacetime of the connection discussed in (3.4). Substituting (4.16) into the gauged action gives the action for the nonlinear $\sigma$-model with target space $F_{0}$ :

$$
S=-\frac{1}{2} \int d^{D} x\left(g_{i j}-g_{i m} g_{j n} k_{A}^{m} k_{B}^{n} H^{A B}\right) \partial^{a} \varphi^{i} \partial_{a} \varphi^{j} .
$$

(D) $C P(1)$

To illustrate the previous discussion we consider the $C P(1)$-model. This model exhibits two features characteristic of supersymmetric constructions: Nonlinear constraints are imposed by Lagrange multipliers and the group action is extended to its complexification.

We start with the target space $S^{3}$ : We use complex coordinates $z^{p}, p=1,2$, constrained by

$$
\sum_{p}\left|z^{p}\right|^{2} \equiv|z|^{2}=1
$$

The Lagrangian $L_{z}$ is

$$
L_{z}=-\left|\partial_{a} z\right|^{2} .
$$

The constraint (4.18) induces the metric on $S^{3}$ [cf. (4.2)]. The Lagrangian is invariant under an obvious global $U(2)$ acting linearly on $z^{p}$ and hence, through (4.18), nonlinearly on the coordinates of $S^{3}$. The full isometry group of $S^{3}$ is $\mathrm{SO}(4)$, but $U(2)$ is the maximal subgroup that acts analytically on $z^{p}$. To construct the $C P(1)$-model we gauge the diagonal $U(1)$-subgroup in $U(2)$. Under this $U(1)$ the coordinates transform as

$$
\delta z^{p}=i \lambda z^{p}, \quad \delta \bar{z}^{\bar{p}}=-i \lambda \bar{z}^{\bar{p}}, \quad \lambda \text { real },
$$

and using (4.14) the gauged Lagrangian is

$$
\tilde{L}_{z}=-\left|\left(\partial_{a}+i A_{a}\right) z\right|^{2}=L_{z}+i A_{a}\left(\bar{z}^{\bar{p}} \overleftrightarrow{\partial}^{a} z^{p}\right)-A^{a} A_{a},
$$

where we have used (4.18) and $\bar{z} \overleftrightarrow{\partial} z \equiv \bar{z} \partial z-z \partial \bar{z}$. Extremizing the action we find the gauge connection

$$
A_{a}=\frac{i}{2}\left(\bar{z}^{\bar{p}} \overleftrightarrow{\partial}_{a} z^{p}\right) .
$$

Substituting (4.22) into $\widetilde{L}_{z}$ we find

$$
L_{0}=L_{z}-\frac{1}{4}\left(\bar{z}^{\bar{p}} \overleftrightarrow{\partial}_{a} z^{p}\right)^{2} .
$$

This Lagrangian is invariant under local U(1)-transformations (4.20), which can be used, e.g., to make $z^{1}$ real. The constraint (4.18) induces the metric on the orbit space $C P(1)$. 
The constraint (4.18) is awkward as it makes the action of the $U(2)$ on $S^{3}$ nonlinear. It is useful to relax the constraint and impose it through a Lagrange multiplier $Y$ in the Lagrangian $L_{0}$ :

$$
L_{0} \rightarrow L_{0}+Y\left(|z|^{2}-1\right) .
$$

As the theory is now described in terms of unconstrained complex fields $z^{p}$ it is natural to extend the $U(1)$-transformation (4.20) to the complexified transformations:

$$
\delta z^{p}=i \Lambda z^{p}, \quad \delta \bar{z}^{\bar{p}}=-i \bar{\Lambda} \bar{z}^{\bar{p}}, \quad \Lambda \text { complex } .
$$

To make the Lagrangian invariant under (4.25) we introduce a new real field $v$ with transformation

$$
\delta v=\frac{i}{2}(\Lambda-\bar{\Lambda})
$$

The fields $e^{-v} z^{i}$ and $e^{-v} \bar{z}^{i}$ then transform under the complexified $U(1)$ with the real parameter $\lambda=\operatorname{Re} \Lambda$ [cf. (4.20)]. Substituting $z^{i} \rightarrow e^{-v} z^{i}$ and $\bar{z}^{i} \rightarrow e^{-v} \bar{z}^{i}$ into the Lagrangian (4.24) we find

$$
L_{v}=-\left|\partial_{a}\left(e^{-v} z\right)\right|^{2}-\frac{1}{4} e^{-4 v}(\bar{z} \overleftrightarrow{\partial z})^{2}+Y e^{-2 v}\left(|z|^{2}-e^{2 v}\right) .
$$

In this form, the constraint imposed by the Lagrange multiplier can be solved for $v$ in a manifestly $U(2)$-covariant way: $e^{2 v}=|z|^{2}$. The Lagrangian (4.27) is a function of the complex variables $z^{1}, z^{2}$ and is invariant under local complex rescalings [cf. (4.25)]. It thus describes $C P(1)$. Substituting for $v$, the Lagrangian becomes

$$
L=-\frac{1}{|z|^{2}}\left(\delta^{p q}-\frac{z^{p} \bar{z}^{\bar{q}}}{|z|^{2}}\right) \partial^{a} \bar{z}^{\bar{p}} \partial_{a} z^{q}
$$

which gives the standard metric on $C P(1)$ in the gauge $z^{1}=1$.

\section{Supersymmetry}

In this section we attempt to introduce supersymmetry to mathematicians in a selfcontained and, hopefully, pedagogical way. For reviews of supersymmetry see, e.g., $[23,24]$. In particular we present the supersymmetric versions of the nonlinear $\sigma$-models of Sect. 4 and material needed for their gauging. This section should make it possible to understand the constructions of the hyperkähler manifolds in Sect. 6.

\section{(A) Introduction}

A supersymmetry algebra is a graded version of the Poincare algebra. It appends to the even generators of space-time motions $P, J$ odd generators $Q$, which transform as spinors of the Lorentz group (generated by $J$ ). The simple supersymmetry algebra in $D$ dimensions has one odd charge; the $N$-extended algebra has $N$ such charges. The structure of the algebra is

$$
\begin{array}{ccc}
{[P, P]=0,} & {[J, J] \sim J,} & {[P, J] \sim P,} \\
{[J, Q] \sim Q,} & {[P, Q]=0,} & \{Q, Q\} \sim P,
\end{array}
$$


where $[$,$] is the usual antisymmetric Lie-bracket and \{$,$\} is a symmetric (super)$ Lie-bracket. The interesting feature is the creature $Q$ : It generates an internal symmetry, cf. (4.6), but it transforms under rotations $J$, and hence $Q$ mixes different spins, i.e., different representations of the Lorentz group. Supersymmetry therefore relates wave operators (and their spectra) for different spins, e.g., Laplace and Dirac operators. The even part (i.e., $P, J$ ) of the algebra in (5.1) is an ordinary Liealgebra: The isometry group for flat space. Other superalgebras, based on larger even algebras, have been studied. These can all be viewed as subalgebras of the graded version of the conformal algebra, and include supersymmetry on a hyperboloid and flat space-time supersymmetry with central extensions.

Here we focus on Poincaré supersymmetry in three space-time dimensions. The detailed superalgebra is given below. To find off-shell (cf. Sect. 4) representations of supersymmetry we introduce superspace as the coset space (superPoincaré group)/(Lorentz group). This has coordinates $x, \theta$, where $x$ are even and $\theta$ odd, thus reflecting the properties of the generators $P$ and $Q$. The group action on the coset space gives a representation of the generators of the superalgebra as differential operators. This realizes supersymmetry transformations as rotations and translations in superspace. Representations of supersymmetry are obtained as fields over superspace, superfields. We assume analyticity in $\theta$ and can define a superfield by its Taylor expansion in $\theta$. This has only a finite number of terms as $\theta$ is nilpotent. The $x$-dependent coefficients in the $\theta$-expansion are called components of the superfield. Each one of them is a representation of the Lorentz group while the entire set of components (the multiplet) is a representation of supersymmetry. This is an off-shell representation, i.e., the component fields do not have to fulfill field equations (cf. Sect. 4).

Superfields are in general reducible representations of supersymmetry. However, irreducible representations can be constructed by imposing constraints on the superfields using a spinorial derivative $D$ that anticommutes with the generator $Q$.

After discussing various $N=1,2$ superfields as fields over $N=1,2$ superspace respectively, we describe $N=2$ supersymmetry in $N=1$ superspace. This amounts to expanding the $N=2$ superfields in half of the $\theta$ 's with coefficients that are functions of the remaining $\theta$ 's (and $x$ ). Each one of these coefficients is a representation of $N=1$ supersymmetry while the entire set of coefficients is a representation of $N=2$ supersymmetry (cf., the components of a superfield above). This technique has several advantages; one is that $N=2$ superfields restrict one to holomorphic coordinates on the target manifold of nonlinear $\sigma$-models, whereas $N=1$ superfields allow arbitrary coordinates. For $N=4$ supersymmetry a finite dimensional description of one of the representations needed for the nonlinear $\sigma$-model is not known in $N=4$ superspace (see footnote 4 in Sect. $5 \mathrm{M}$ below), and we use $N=1,2$ superspace.

We introduce action functionals in superspace [cf. (4.1)] that are invariant (up to a boundary term) under supersymmetry transformations and give the action functional for the $N=1$ supersymmetric nonlinear $\sigma$-model in superspace. Expanding in components we find that this is an extension of (4.2). The target manifold is a general Riemannian manifold. If the action functional is required to be invariant under additional supersymmetries, it is found that it is $N=2$ 
supersymmetric if and only if the target manifold is Kähler and $N=4$ supersymmetric if and only if the target manifold is hyperkähler.

\section{(B) Preliminaries}

Our notation follows essentially that of [24]. In three dimensional space-time (with signature -++ ) the Lorentz group is $S L(2, R)$ and the corresponding fundamental representation acts on real (Majorana) two-component spinors $\psi^{\alpha}=\left(\psi^{+}, \psi^{-}\right)$. We use spinor notation for all Lorentz representations, denoting spinor indices by Greek letters. Thus vectors (the three-dimensional representation) are described by symmetric second-rank spinors $V^{a} \leftrightarrow V^{\alpha \beta}=\left(V^{++}, V^{+-}\right.$, $V^{--}$) or, equivalently, traceless second rank spinors $V_{\alpha}^{\beta}$. All our odd rank spinors are anticommuting (Grassman).

Spinor indices are raised and lowered by the second-rank antisymmetric symbol $C_{\alpha \beta}$, which also defines the spinor inner product:

$$
\begin{aligned}
C_{\alpha \beta} & =-C_{\beta \alpha}=-C^{\alpha \beta}=\left(\begin{array}{cc}
0 & -i \\
i & 0
\end{array}\right), \\
C_{\alpha \beta} C^{\gamma \delta} & =\delta_{[\alpha}^{\gamma} \delta_{\beta]}^{\delta} \equiv \delta_{\alpha}^{\gamma} \delta_{\beta}^{\delta}-\delta_{\beta}^{\gamma} \delta_{\alpha}^{\delta}, \\
\psi_{\alpha} & =\psi^{\beta} C_{\beta \alpha}, \quad \psi^{\alpha}=C^{\alpha \beta} \psi_{\beta}, \\
\psi \chi & =\psi^{\alpha} \chi_{\alpha}=\chi^{\alpha} \psi_{\alpha}=i\left(\chi^{+} \psi^{-}-\chi^{-} \psi^{+}\right) .
\end{aligned}
$$

Note that we (anti)symmetrize without a combinatorial factor. We often use the identity

$$
A_{[\alpha} B_{\beta]}=-C_{\alpha \beta} A^{\gamma} B_{\gamma},
$$

which follows from (5.2). We use $C_{\alpha \beta}$ (instead of the real $\varepsilon_{\alpha \beta}$ ) to simplify the rules for hermitian conjugation. In particular, it makes $\psi^{\alpha} \psi_{\alpha}$ hermitian (recall $\psi^{\alpha}$ and $\psi_{\alpha}$ anticommute). Note however that whereas $\psi^{\alpha}$ is real, $\psi_{\alpha}$ is imaginary.

\section{(C) Superalgebras and Superspace}

The $N$-extended superalgebra in three dimensions is

$$
\begin{aligned}
& {\left[P_{\alpha \beta}, P_{\gamma \delta}\right]=0, \quad\left[J_{\alpha \beta}, J^{\gamma \delta}\right]=-\frac{i}{2} \delta_{(\alpha}^{\left(\gamma J_{\beta}^{\delta}\right)},} \\
& {\left[J_{\alpha \beta}, P^{\gamma \delta}\right]=-\frac{i}{2} \delta_{(\alpha}^{(\gamma} P_{\beta)}^{\delta)},} \\
& {\left[Q_{\alpha}^{i}, P_{\beta \gamma}\right]=0, \quad\left[J_{\alpha \beta}, Q_{\gamma}^{i}\right]=\frac{i}{2} C_{\gamma(\alpha} Q_{\beta)}^{i},} \\
& \left\{Q_{\alpha}^{i}, Q_{\beta}^{j}\right\}=2 P_{\alpha \beta} \delta^{i j} .
\end{aligned}
$$

Here, $i, j=1, \ldots, N$ label the odd generators; $P_{\alpha \beta}=P_{\beta \alpha} \leftrightarrow P_{a}$ are the generators of translations and $J_{\alpha \beta}=J_{\beta \alpha} \leftrightarrow J_{a}=\frac{1}{2} \varepsilon_{a b c} J^{b c}$ generate rotations.

A convenient way of obtaining off-shell representations of supersymmetry is to use superspace. Ordinary space-time can be defined as the coset space (Poincaré 
group)/(Lorentz group). Similarly, superspace can be defined as (super-Poincaré group)/(Lorentz group). We parametrize this coset space as

$$
h(x, \theta)=e^{i\left(x^{\alpha \beta} P_{\alpha \beta}+\theta^{i \alpha} Q_{\alpha}^{i}\right)} .
$$

The parameters $x$ and $\theta$ are the coordinates of superspace. Reflecting the grading of the generators $P$ and $Q, x$ takes its values in the even part of a Grassman algebra and $\theta$ in the odd part:

$$
[x, x]=[x, \theta]=\{\theta, \theta\}=\{\theta, Q\}=[\theta, P]=[x, P]=0 .
$$

We find a representation $R$ of a super-Poincaré group element $g$ by letting it act on superspace as

Considering

$$
h(R x, R \theta) \equiv g^{-1} h(x, \theta) \bmod (\text { Lorentz group }) \text {. }
$$

$$
g_{\omega}=e^{-i \omega_{\alpha}^{\beta} J_{\beta}^{\alpha}}, \quad g_{\xi}=e^{-i \xi^{\alpha \beta} P_{\alpha \beta}}, \quad g_{\varepsilon}=e^{-i \varepsilon^{i \alpha} Q_{\alpha}^{i}},
$$

respectively, we find, using the Baker-Hausdorff formula $e^{A} e^{B}=e^{A+B+\frac{1}{2}[A, B]}$ when $[A, B]$ commutes with $A$ and $B$ :

$$
\begin{aligned}
& {\left[R\left(g_{\omega}\right) x\right]^{\alpha \beta}=\left(e^{\omega}\right)_{\gamma}{ }^{\alpha}\left(e^{\omega}\right)_{\delta}{ }^{\beta} x^{\gamma \delta},} \\
& {\left[R\left(g_{\omega}\right) \theta\right]^{i \alpha}=\left(e^{\omega}\right)_{\beta}{ }^{\alpha} \theta^{i \beta},} \\
& {\left[R\left(g_{\xi}\right) x\right]^{\alpha \beta}=x^{\alpha \beta}+\xi^{\alpha \beta}, \quad\left[R\left(g_{\xi}\right) \theta\right]^{i \alpha}=\theta^{i \alpha},} \\
& {\left[R\left(g_{\varepsilon}\right) x\right]^{\alpha \beta}=x^{\alpha \beta}-\frac{i}{2} \varepsilon^{i(\alpha} \theta^{i \beta)}, \quad\left[R\left(g_{\varepsilon}\right) \theta\right]^{i \alpha}=\theta^{i \alpha}+\varepsilon^{i \alpha} .}
\end{aligned}
$$

Supersymmetry transformations are thus realized as rotations and translations in superspace.

\section{(D) Superfields and Spinor Derivatives}

We define superfields as a generalization of the fields defined in Sect. 4. Thus a scalar superfield $\Phi(x, \theta)$ is a map from superspace into the even part of a Grassman algebra; a spinor superfield $\Psi_{\alpha}(x, \theta)$ is a space-time spinor with values in the odd part of a Grassman algebra. The $\theta$-dependence in a superfield is formal: We assume analyticity in $\theta$, which, because of the nilpotency of $\theta$, implies that a superfield is a polynomial in $\theta$. We can find representations of the supersymmetry algebra acting on superfields $\Phi$ by defining these fields to transform as coordinate scalars under (5.9):

$$
g \Phi(R(g) x, R(g) \theta)=\Phi(x, \theta)
$$

or, infinitesimally,

$$
\delta \Phi=-i\left[\xi^{\alpha \beta} P_{\alpha \beta}+\omega_{\beta}^{\alpha} J_{\alpha}^{\beta}+\varepsilon^{i \alpha} Q_{\alpha}^{i}, \Phi\right] .
$$

From (5.9) we find the representation of the generators as differential operators:

$$
\begin{aligned}
& r(P)=i \partial_{\alpha \beta}, \\
& r(J)=-\frac{i}{2}\left(x_{(\alpha}^{\gamma} \partial_{\beta) \gamma}+\theta_{(\alpha}^{i} \partial_{\beta)}^{i}\right), \\
& r(Q)=i \partial_{\alpha}^{i}+\theta^{i \beta} \partial_{\alpha \beta} .
\end{aligned}
$$


Here $\partial_{\alpha}^{i}$ is the anticommuting partial $\theta$-derivative: $\partial_{\alpha}^{i} \theta^{j \beta}=\delta^{i j} \delta_{\beta}^{\alpha}$. As everything is polynomial in $\theta$ this algebraic definition is adequate. In general, when acting on spinor superfields, etc., we must add a matrix representation of the Lorentz group to $r(J)$, e.g.,

$$
r(J) \Psi_{\gamma}=-\frac{i}{2}\left[x_{(\alpha}^{\delta} \partial_{\beta) \delta}+\theta_{(\alpha}^{i} \partial_{\beta)}^{i}\right] \Psi_{\gamma}-\frac{i}{2} C_{\gamma(\alpha} \Psi_{\beta)} .
$$

Given a superfield that transforms covariantly, i.e., according to (5.10-13), we want to define differentiation that preserves the transformation properties. In (flat) space-time $\partial_{\alpha \beta}$ is such a derivative; it transforms covariantly in (flat) superspace as well. However, the spinorial derivatives $\partial_{\alpha}^{i}$ do not transform covariantly [they do not anticommute with $r(Q)]$. The $r(Q)$ was induced by left multiplication (5.7); right multiplication gives the spinorial derivative $D_{\alpha}^{i}$

$$
D_{\alpha}^{i}=\partial_{\alpha}^{i}+i \theta^{i \beta} \partial_{\alpha \beta},
$$

which anticommutes with $r(Q)$. The $D$ 's obey an algebra isomorphic to the algebra of the $Q$ 's:

$$
\left\{D_{\alpha}^{i}, D_{\beta}^{j}\right\}=2 i \partial_{\alpha \beta} \delta^{i j} .
$$

Superfields are in general reducible representations of supersymmetry. A systematic construction of the irreducible representations is possible using the spinor derivatives $D_{\alpha}^{i}$. Here we give a heuristic discussion of the simplest $N=1,2$ superfields in three dimensions. When discussing $N=4$ supersymmetry we do not use $N=4$ superspace, but instead we use representations in terms of $N=1,2$ superfields (see below).

\section{(E) $N=1$ Scalar Superfield}

The $N=1$ real scalar superfield $\Phi$ is an irreducible representation of $N=1$ supersymmetry. To make contact with formulations in ordinary space-time we need to relate superfields to ordinary space-time fields. As mentioned above, a superfield is polynomial in $\theta$. The space-time dependent coefficients in this expansion are the ordinary fields. Instead of doing the Taylor expansion of a superfield using the partial derivative $\partial_{\alpha}$, we use the spinorial derivative $D_{\alpha}$. We define the components of $\Phi$ as $\theta$-independent parts of $\Phi$ and of spinor derivatives of $\Phi$ :

$$
A=\Phi\left|, \quad \psi_{\alpha}=D_{\alpha} \Phi\right|, \quad F=\frac{1}{2} D^{2} \Phi \mid,
$$

here $\mid$ denotes the $\theta$-independent part. Higher order spinor derivatives lead only to space-time derivatives of the components in (5.16) because, for $N=1$,

$$
\left\{D_{\alpha}, D_{\beta}\right\}=2 i \partial_{\alpha \beta},
$$

which implies

$$
\begin{gathered}
D_{\alpha} D_{\beta}=i \partial_{\alpha \beta}-\frac{1}{2} C_{\alpha \beta} D^{2}, \quad D^{\alpha} D_{\beta} D_{\alpha}=0, \\
D^{2} D_{\alpha}=-D_{\alpha} D^{2}=2 i \partial_{\alpha \beta} D^{\beta} .
\end{gathered}
$$

The superfield $\Phi$ contains a multiplet of component fields: $A$ is a real scalar (in ordinary space-time), $\psi_{\alpha}$ is a spinor and $F$ is another real scalar. We will see later that $A$ and $F$ play very different roles. 
(F) $N=1$ Gauge Superfield

The other $N=1$ superfield we use is a real gauge spinor potential $\Gamma_{\alpha}$. This contains a component vector gauge potential (see below) and hence enters both in the Legendre construction (a vector is dual to a scalar in three dimensions) and in the quotient construction. In the abelian case the gauge transformation is

$$
\delta \Gamma_{\alpha}=-i D_{\alpha} X,
$$

where $X$ is a real scalar superfield [cf. (5.16)]. From $\Gamma_{\alpha}$ we can construct a vector potential

$$
\Gamma_{\alpha \beta}=-\frac{i}{2} D_{(\alpha} \Gamma_{\beta)}
$$

transforming as

$$
\delta \Gamma_{\alpha \beta}=-i \partial_{\alpha \beta} X
$$

and a divergence free gauge invariant spinor field strength

$$
F_{\alpha}=i D^{\beta} D_{\alpha} \Gamma_{\beta}, \quad D^{\alpha} F_{\alpha}=0
$$

[invariance follows from (5.18)]. The usual vector field strength $F_{\alpha \beta} \leftrightarrow F_{a}=\varepsilon_{a b c} \partial_{b} \Gamma_{c}$ can be found from $F_{\alpha}$,

$$
F_{\alpha \beta}=i D_{(\alpha} F_{\beta)} \sim \partial_{(\alpha}^{\gamma} \Gamma_{\beta) \gamma} .
$$

The components can be obtained by differentiation and projection onto the $\theta$-independent sector as for $\Phi$. The gauge invariant components are obtained from $F_{\alpha}$

$$
\lambda_{\alpha}=F_{\alpha}\left|, \quad f_{\alpha \beta}=i D_{(\alpha} F_{\beta)}\right|=F_{\alpha \beta} \mid .
$$

Because $D^{\alpha} F_{\alpha}=0$, there is no independent $D^{2} F \mid$ component $\left(D^{2} F_{\alpha} \mid=+2 i \partial_{\alpha}^{\beta} \lambda_{\beta}\right)$.

The potential $\Gamma_{\alpha}$ has a natural interpretation as the spinor component of a connection super 1-form $\Gamma$,

$$
\Gamma=\Gamma_{\alpha} d \theta^{\alpha}+\Gamma_{\alpha \beta} d x^{\alpha \beta},
$$

$\left[\Gamma_{\alpha \beta}, \Gamma_{\alpha}\right.$ are superfields; the notion of components used here must not be confused with the components of a superfield discussed above, cf. (5.16).] The connection can be used to define a gauge covariant derivative $\nabla_{A}=\left(\nabla_{\alpha}, \nabla_{\alpha \beta}\right)$

$$
\nabla_{\alpha}=D_{\alpha}+\Gamma_{\alpha}, \quad \nabla_{\alpha \beta}=\partial_{\alpha \beta}+\Gamma_{\alpha \beta} .
$$

It satisfies the algebra

$$
\left\{\nabla_{\alpha}, \nabla_{\beta}\right\}=2 i \nabla_{\alpha \beta},
$$

cf. (5.17), and

$$
\begin{aligned}
{\left[\nabla_{\alpha}, \nabla_{\beta \gamma}\right] } & =-\frac{1}{2} C_{\alpha(\beta} F_{\gamma)}, \\
{\left[\nabla_{\alpha \beta}, \nabla_{\gamma \delta}\right] } & =\frac{1}{2}\left(C_{\alpha(\gamma} F_{\delta) \beta}+C_{\beta(\delta} F_{\gamma) \alpha}\right),
\end{aligned}
$$

where the curvatures $F_{\alpha}, F_{\alpha \beta}$ are the spinor and vector field strengths defined in $(5.22,23)$. Note that $(5.27)$ expresses the vector connection $\Gamma_{\alpha \beta}$ in terms of the 
spinor connection $\Gamma_{\alpha}$, cf. (5.20), and that (5.28) follows from (5.27) via the super Bianchi identity,

$$
0=(-)^{A C}\left[\left[\nabla_{A}, \nabla_{B}\right\}, \nabla_{C}\right\}+(-)^{A B}\left[\left[\nabla_{B}, \nabla_{C}\right\}, \nabla_{A}\right\}+(-)^{B C}\left[\left[\nabla_{C}, \nabla_{A}\right\}, \nabla_{B}\right\},
$$

where the super bracket is

$$
\left[\nabla_{A}, \nabla_{B}\right\}=\nabla_{A} \nabla_{B}-(-)^{A B} \nabla_{B} \nabla_{A},
$$

and $(-)^{A B}=-1$ if $A=\alpha, B=\beta$ and $(-)^{A B}=+1$ otherwise.

The nonabelian case is defined by (5.26-28) where the connections and curvatures are Lie-algebra valued.

\section{(G) $N=1$ Supersymmetry Transformations}

From the transformations (5.11) we find the supersymmetry transformations of the component fields. For a scalar superfield we find

$$
\begin{aligned}
\delta_{Q} A & =-\varepsilon^{\alpha} \psi_{\alpha}, \\
\delta_{Q} \psi_{\alpha} & =\varepsilon_{\alpha} F-i \varepsilon^{\beta} \partial_{\alpha \beta} A, \\
\delta_{Q} F & =-i \varepsilon^{\beta} \partial_{\beta}^{\alpha} \psi_{\alpha} .
\end{aligned}
$$

The transformation of $F$ has the form of a Dirac-operator acting on $\psi_{\alpha}$. This is a general feature of supersymmetry: The highest component (in the $\theta$-expansion) always transforms as a Dirac-operator acting on the second highest component.

The supersymmetry transformations relate the wave operators (and their spectra) for the component fields in a superfield. For example, for a scalar superfield the free (i.e., linear) field equations are:

$$
F=m A, \quad i \partial_{\beta}^{\alpha} \psi^{\beta}=-m \psi^{\alpha}, \quad \frac{1}{2} \square A=m F, \quad \square \equiv \partial^{\alpha \beta} \partial_{\alpha \beta} .
$$

These equations rotate into each other under supersymmetry. This holds also when nonlinear terms are present. Note that the $F$-field equation is algebraic. $F$ is an auxiliary field that is needed for the closure of the algebra [off-shell representation, cf. discussion following (4.6)]: When it is eliminated through its field equation, the algebra closes on the remaining fields only if they satisfy their field equations (on-shell representation) (actually, it is sufficient that the spinor field equation is satisfied). An important feature of superspace is that superfields provide auxiliary fields for off-shell closure of the algebra.

\section{(H) $N=2$ Spinor Derivatives and Superfields}

For $N=2$ the situation is considerably more involved: A scalar superfield decomposes into three irreducible projections. We define complex spinor derivatives

$$
D_{\alpha} \equiv \frac{1}{2}\left(D_{\alpha}^{1}+i D_{\alpha}^{2}\right), \quad \bar{D}_{\alpha} \equiv \frac{1}{2}\left(D_{\alpha}^{1}-i D_{\alpha}^{2}\right),
$$

with the algebra

$$
\left\{D_{\alpha}, \bar{D}_{\beta}\right\}=i \partial_{\alpha \beta}, \quad\left\{D_{\alpha}, D_{\beta}\right\}=\left\{\bar{D}_{\alpha}, \bar{D}_{\beta}\right\}=0,
$$


which implies

$$
\left[D_{\alpha}, \bar{D}^{2}\right]=-2 i \bar{D}^{\beta} \partial_{\alpha \beta}, \quad D^{\alpha} \bar{D}_{\alpha}=\bar{D}^{\alpha} D_{\alpha}, \quad\left(D^{\alpha} \bar{D}_{\alpha}\right)^{2}=-\frac{1}{2} D^{\alpha} \bar{D}^{\beta} \bar{D}_{\beta} D_{\alpha}
$$

For a scalar superfield we can impose a covariant constraint

which implies, because $\bar{D}^{3}=0$,

$$
\bar{D}_{\alpha} \Phi=0,
$$

$$
\Phi=\bar{D}^{2} \Psi
$$

for arbitrary $\Psi . \Phi$ is called a chiral superfield and is irreducible. ( $\Phi$ must be complex, otherwise $\bar{D} \Phi=D \Phi=0 \Rightarrow\left\{D_{\alpha}, \bar{D}_{\beta}\right\} \Phi=i \partial_{\alpha \beta} \Phi=0$.) Another characterization of a chiral superfield $\Phi$ is [as follows from (5.34-36)]

$$
\frac{1}{2} \bar{D}^{2} D^{2} \Phi=\square \Phi .
$$

The other irreducible representation we use is the real linear superfield $G$ which obeys

$$
D^{2} G=\bar{D}^{2} G=0,
$$

or, equivalently

$$
D^{\alpha} \bar{D}^{2} D_{\alpha} G=-\square G,
$$

which implies

$$
G=D^{\alpha} \bar{D}_{\alpha} V
$$

for arbitrary real $V$. The superfields $\Phi, \bar{\Phi}\left(D_{\alpha} \bar{\Phi}=0\right)$ and $G$ are the complete decomposition into irreducible parts of scalar superfields because of $(5.38,40)$ and

$$
\frac{1}{2} D^{2} \bar{D}^{2}-D^{\alpha} \bar{D}^{2} D_{\alpha}+\frac{1}{2} \bar{D}^{2} D^{2}=\square .
$$

\section{(I) $N=2$ Components and Supersymmetry Transformations}

We define the components of a chiral superfield $\Phi$ as the $\theta$-independent parts of $\Phi$ and its complex spinor derivatives:

$$
A=\Phi\left|, \quad \psi_{\alpha}=D_{\alpha} \Phi\right|, \quad F=\frac{1}{2} D^{2} \Phi \mid .
$$

$\bar{D}_{\alpha} \Phi=0$ because $\Phi$ is chiral and from $(5.34,35)$ we see that all mixed $D, \bar{D}$ components of $\Phi$ are expressible as spacetime derivatives of the components in (5.43). The chiral superfield $\Phi$ contains a multiplet of component fields: $A$ is a complex scalar, $\psi_{\alpha}$ is a complex spinor and $F$ is another complex scalar. Note that an $N=2$ chiral superfield is just a complex version of an $N=1$ real superfield [cf. (5.16)], which is why the chiral superfields appear in $N=2$ nonlinear $\sigma$-models and why these have a natural complex structure. A real scalar superfield has the components

$$
\begin{aligned}
& C=V\left|, \quad \chi_{\alpha}=i D_{\alpha} V\right|, \quad \bar{\chi}_{\alpha}=-i \bar{D}_{\alpha} V \mid, \\
& M=\frac{1}{2} D^{2} V\left|, \quad \bar{M}=\frac{1}{2} \bar{D}^{2} V\right|, \quad A_{\alpha \beta}=\frac{1}{4}\left[\bar{D}_{(\alpha}, D_{\beta)}\right] V \mid, \\
& A=\bar{D}^{\alpha} D_{\alpha} V\left|, \quad \lambda_{\alpha}=\frac{i}{2} \bar{D}^{2} D_{\alpha} V\right|, \quad \bar{\lambda}_{\alpha}=-\frac{i}{2} D^{2} \bar{D}_{\alpha} V \mid, \\
& E=\frac{1}{4} D^{\alpha} \bar{D}^{2} D_{\alpha} V \mid .
\end{aligned}
$$


We emphasize that $V$ contains a vector $A_{\alpha \beta}$ and that it is a reducible superfield [see (5.42)]. For the particular case when $V=G$ is linear we find

$$
M=0, \quad \partial^{\alpha \beta} A_{\alpha \beta}=0, \quad \lambda_{\alpha}=-i \partial_{\alpha \beta} \bar{\chi}^{\beta}, \quad E=-\frac{1}{4} \square C,
$$

leaving

$$
C=G\left|, \quad \chi_{\alpha}=i D_{\alpha} G\right|, \quad A_{\alpha \beta}=\frac{1}{4}\left[\bar{D}_{(\alpha,}, D_{\beta)}\right] G\left|, \quad A=\bar{D}^{\alpha} D_{\alpha} G\right|
$$

as the components in $G$. From the transformation (5.11) we find the $N=2$ supersymmetry transformations of the component fields. For example, the components of the chiral superfield transform as

$$
\delta_{Q} A=-\varepsilon^{\alpha} \psi_{\alpha}, \quad \delta_{Q} \psi_{\alpha}=\varepsilon_{\alpha} F-i \bar{\varepsilon}^{\beta} \partial_{\alpha \beta} A, \quad \delta_{Q} F=-i \bar{\varepsilon}^{\beta} \partial_{\beta}^{\alpha} \psi_{\alpha} .
$$

\section{(J) $N=2$ Gauge Fields}

We now consider $N=2$ gauge fields. We expect real scalar superfields to enter, as they contain vector components $A_{\alpha \beta}(5.44)$. As for the nonabelian $N=1$ gauge spinor we define the $N=2$ gauge multiplet by giving the algebra of its covariant derivatives:

$$
\begin{aligned}
& \left\{\nabla_{\alpha}, \nabla_{\beta}\right\}=\left\{\bar{\nabla}_{\alpha}, \bar{\nabla}_{\beta}\right\}=0, \\
& \left\{\nabla_{\alpha}, \bar{\nabla}_{\beta}\right\}=i \nabla_{\alpha \beta}+C_{\alpha \beta} F .
\end{aligned}
$$

Equation (5.49) defines the vector connection in terms of the spinor connection [cf., the $N=1$ case (5.27)]. $F$ is the fundamental field strength; in the abelian case the Bianchi identities imply that it is linear $(5.39-41,46)$ (in the nonabelian case it is covariantly linear $\nabla^{\alpha} \nabla_{\alpha} F=\bar{\nabla}^{\alpha} \bar{\nabla}_{\alpha} F=0$ ). The Bianchi identities give the remaining graded commutators where the field strengths are covariant derivatives of $F$, e.g., $F_{\alpha}=\nabla_{\alpha} F[\mathrm{cf} .(5.28)]$.

Equation (5.48) is a nontrivial constraint on the spinor connection; it has no analog in the $N=1$ case. It is the integrability condition for the existence of covariantly chiral superfields

$$
\bar{\nabla}_{\alpha} \Phi=0 \Rightarrow\left\{\bar{\nabla}_{\alpha}, \bar{\nabla}_{\beta}\right\} \Phi=0 .
$$

A particular solution to the constraint (5.48) is

$$
\nabla_{\alpha}=e^{-V} D_{\alpha} e^{V}, \quad \bar{\nabla}_{\alpha}=\bar{D}_{\alpha},
$$

where $V=V^{A} i T_{A}$ is a hermitian Lie-algebra valued scalar superfield. The most general solution is found from (5.51) by an arbitrary complexified gauge transformation

$$
\nabla_{\alpha}=e^{-W} e^{-V} D_{\alpha} e^{V} e^{W}, \quad \bar{\nabla}_{\alpha}=e^{-W} \bar{D}_{\alpha} e^{W} .
$$

The solution (5.51) is called chiral representation and is not hermitian; (5.52) allows for hermitian solutions (choose $e^{V} e^{W}=e^{-\bar{W}}$ ). Clearly, we can also use (5.52) to find an antichiral representation (choose $W=-V$ )

$$
\nabla_{\alpha}=D_{\alpha}, \quad \bar{\nabla}_{\alpha}=e^{V} \bar{D}_{\alpha} e^{-V} .
$$




\section{(K) $N=2$ Gauge Transformations}

We now consider the transformations of chiral superfields. The most natural local (i.e., superspace dependent, see Sect. 4A) representation of a Lie-group on chiral superfields $\Phi$ is with chiral group elements $e^{i \Lambda}$,

$$
\begin{gathered}
g(\Lambda) \Phi=e^{i \Lambda} \Phi, \quad \Lambda=i \Lambda^{A} T_{A}, \\
\bar{D}_{\alpha} \Lambda=0,
\end{gathered}
$$

where $T_{A}$ is the appropriate matrix representation of the generators acting on the vector space of $\Phi$ 's. Recall that, because $\Lambda$ is chiral and non-constant, it must be complex $\Lambda \neq \bar{\Lambda}$. The antichiral superfield $\bar{\Phi}$ transforms with $\bar{\Lambda}$

$$
g(\bar{\Lambda}) \bar{\Phi}=\bar{\Phi} e^{-i \bar{\Lambda}}, \quad \bar{\Lambda}=i \bar{\Lambda}^{A} T_{A}, \quad D_{\alpha} \bar{\Lambda}=0 .
$$

This means that the group naturally acts on chiral superfields through its complexification. The covariant derivative can be chosen to transform covariantly with respect to $\Lambda$ or $\bar{\Lambda}$ but not with respect to both. The $\Lambda$-covariant transformation follows from (5.54)

$$
g(\Lambda) \nabla_{A}=e^{i \Lambda} \nabla_{A} e^{-i \Lambda} .
$$

Because $\Lambda$ is chiral, this is compatible with the chiral representation (5.51) if we let $V$ transform as

$$
g(\Lambda) e^{V}=e^{i \bar{\Lambda}} e^{V} e^{-i \Lambda} .
$$

The $\bar{A}$-covariant transformations which follow from (5.56) are compatible with the antichiral representation (5.53) with $V$ transforming as in (5.58).

\section{(L) $N=1$ Form of $N=2$ Supersymmetry}

We formulate $N=2$ supersymmetry in terms of $N=1$ superfields because $N=2$ superfields restrict one to holomorphic coordinates on the target space for nonlinear $\sigma$-models, whereas $N=1$ superfields allow arbitrary coordinates. Expressing $N=2$ superfields in terms of $N=1$ superfields is analogous to expressing superfields in terms of components [cf. (5.16)]. This amounts to explicitly expanding an $N=2$ superfield in terms of half of the $\theta$ 's with coefficients that depend on the rest of the $\theta$ 's (and $x$ ), and hence are $N=1$ superfields. Technically, we split the $N=2$ spinorial derivative $D_{\alpha}$ into an $N=1$ spinorial derivative $\check{D}_{\alpha}$ and an orthogonal operator $\check{Q}_{\alpha}$,

$$
\begin{gathered}
\check{D}_{\alpha}=D_{\alpha}+\bar{D}_{\alpha}=D_{\alpha}^{1}, \\
\breve{Q}_{\alpha}=i\left(\bar{D}_{\alpha}-D_{\alpha}\right)=D_{\alpha}^{2} .
\end{gathered}
$$

$\check{D_{\alpha}}$ is the usual $N=1$ derivative obeying the algebra (5.17). In $N=1$ superspace, only one supersymmetry is manifest; the other supersymmetry mixes different superfields [this is analogous to component transformations (5.31)]. The latter supersymmetry is generated by $\breve{Q}$. We also use $\breve{Q}$ to define the $N=1$ superfield components of an $N=2$ superfield.

The discussion above is a sketch of the decomposition of $N=2$ superfields into $N=1$ superfields. Here we give the results for the $N=2$ chiral and gauge multiplets, 
for details see [25]. A model with $p N=2$ chiral superfields decomposes into $2 p$ real scalar $N=1$ superfields $\Phi^{i}$ (the real and imaginary parts). In arbitrary coordinates, the second supersymmetry is

$$
\delta_{\eta} \Phi^{i}=\left(\eta^{\alpha} D_{\alpha} \Phi^{j}\right) \mathbf{I}_{j}^{i} .
$$

The supersymmetry algebra (5.4) requires that I is an integrable complex structure. The $N=2$ (nonabelian) gauge multiplet [cf. $(5.48,49,51,58)]$ decomposes into an $N=1$ gauge multiplet $\Gamma_{\alpha}^{A} T_{A}$ [cf. (5.26)] and a real Lie algebra valued $N=1$ scalar superfield $\Phi=\Phi^{A} T_{A}$ with supersymmetry transformations

$$
\delta \Phi^{A}=\eta^{\alpha} F_{\alpha}^{A}, \quad \delta \Gamma_{\alpha}^{A}=\eta_{\alpha} \Phi^{A},
$$

where $F_{\alpha}^{A}$ is the field strength in (5.28) and $\Phi^{A}$ are coordinates chosen to transform linearly under the gauge transformations (5.54).

\section{(M) $N=4$ Supersymmetry}

We now consider $N=4$ supersymmetry. The off-shell $N=4$ superspace description of $N=4$ gauge multiplets is known; however, such a description is not known for the $N=4$ scalar multiplet needed to describe $N=4$ nonlinear $\sigma$-models ${ }^{4}$. We therefore describe $N=4$ superfields in terms of $N=1,2$ superfields. This also allows a formulation in terms of the Kähler potential.

A model with $k N=4$ scalar multiplets can be described by $4 k$ real scalar $N=1$ superfields $\Phi^{i}$. The three nonmanifest supersymmetries are

$$
\delta_{\eta} \Phi^{i}=\mathbf{I}_{\dot{j}}^{i} \eta_{\mathbf{1}}^{\alpha} D_{\alpha} \Phi^{j}+\mathbf{J}_{\dot{j}}^{i} \eta_{\mathbf{J}}^{\alpha} D_{\alpha} \Phi^{j}+\mathbf{K}_{j}^{i} \eta_{\mathbf{K}}^{\alpha} D_{\alpha} \Phi^{j} .
$$

The supersymmetry algebra (5.4) requires that I, J, K are three integrable complex structures that generate the algebra of the quaternions. The $N=2$ description selects one complex structure, e.g. I, and puts it in canonical form. We can then describe a model with $k N=4$ scalar multiplets in terms of $2 k$ chiral $N=2$ superfields $\Phi^{p}$, where $p$ runs over holomorphic indices only. The two nonmanifest supersymmetries are ( $\eta$ complex) [3]

$$
\delta_{\eta} \Phi^{p}=\bar{D}^{2}\left(\bar{\eta} X^{p}\right),
$$

where $\eta$ is a complex constant chiral superfield parameter satisfying

$$
\bar{D}_{\alpha} \eta=D^{2} \eta=\partial_{a} \eta=0
$$

and $X^{p}(\Phi, \bar{\Phi})$ is (locally) a vector field from which, as a consequence of the supersymmetry algebra (5.4), the integrable complex structures $\mathbf{J}$ and $\mathbf{K}$ are constructed as follows:

$$
\mathbf{J}=\left(\begin{array}{cc}
0 & X^{q}, \bar{p} \\
\bar{X}^{\bar{q}}, p & 0
\end{array}\right), \quad \mathbf{K}=\left(\begin{array}{cc}
0 & i X^{q}, \bar{p} \\
-i \bar{X}^{\bar{q}}{ }_{, p} & 0
\end{array}\right) .
$$

\footnotetext{
${ }^{4}$ This was the case at the time when these lines were written. In the intervening years an interesting off-shell formulation (but with an infinite number of components) of $N=4$ scalar multiplets has been developed [26]. Unfortunately, the problem of extracting the geometry (i.e., the metric) of the $\sigma$-model in this formulation has not been solved
} 
The $N=4$ gauge multiplet is described by an $N=2$ gauge superfield $V=V^{A} T_{A}$ and a Lie algebra valued $N=2$ chiral superfield $\Phi=\Phi^{A} T_{A}$. The two nonmanifest supersymmetries are $[27,5]$

$$
\delta \Phi^{A}=-\left(\bar{\nabla}^{\alpha} F^{A}\right) D_{\alpha} \eta, \quad \delta e^{V}=\bar{\eta} e^{V} \Phi+\eta \bar{\Phi} e^{V},
$$

where $F^{A}$ is the field strength (5.49) and $\eta$ is as above. Whereas the transformation (5.67) generate an off-shell algebra, the algebra of (5.64-66) closes only on fields satisfying the field equations (see below).

\section{(N) Actions in Superspace}

We now turn to action functionals in superspace. Supersymmetrically invariant actions in $N=1$ superspace can be written as

$$
S=\int d^{3} x D^{2} L(\Phi) .
$$

When $L$ is a function of $\Phi$ and its spinorial $(D)$ and spacetime derivatives, it transforms as a superfield, and, in particular, its highest component, $D^{2} L$, transforms into a spacetime divergence [see the discussion following (5.31)]. Discarding this boundary term, the action functional is invariant under supersymmetry.

The action for supersymmetric nonlinear $\sigma$-models is [cf. (4.2)]

$$
S=-\frac{1}{2} \int d^{3} x D^{2}\left(g_{i j}(\Phi) D^{\alpha} \Phi^{i} D_{\alpha} \Phi^{\jmath}\right),
$$

where $g_{i j}(\Phi \mid)$ is a metric on a Riemannian manifold. Using the definitions of the components (5.16) and eliminating the auxiliary field $F$ using its field equation, we find the component action

$$
S=-\frac{1}{2} \int d^{3} x\left[g_{i j}(A)\left(\partial^{\alpha} A^{i} \partial_{a} A^{j}+i \psi_{\alpha}^{i} D^{\alpha \beta} \psi_{\beta}^{j}\right)+\frac{1}{6} R_{i j k \ell} \psi^{\alpha i} \psi_{\alpha}^{k} \psi^{\beta j} \psi_{\beta}^{\ell}\right],
$$

where

$$
\nabla_{\alpha \beta} \psi_{\gamma}^{i} \equiv \partial_{\alpha \beta} \psi_{\gamma}^{i}+\Gamma_{j k}^{i}\left(\partial_{\alpha \beta} A^{j}\right) \psi_{\gamma}^{k} .
$$

Here $\Gamma_{k \ell}^{j}$ and $R_{i j k \ell}$ are the Christoffel connection and Riemann tensor of the target manifold with metric $g_{i j}$. The fields $A^{i}$ are the coordinates of the target manifold, whereas the $\psi_{\alpha}^{i}$ s take their values in the tensor product space of spacetime spinors and tangent vectors at the point $A^{i}$ of the target manifold.

One can also add an arbitrary function of $\Phi^{i}, P\left(\Phi^{i}\right)$, to the Lagrangian (5.69). This adds nonderivative interaction terms.

If we require the action (5.69) to be invariant under extra supersymmetry transformations (5.61), we find that the metric $g$ is hermitian with respect to the complex structure $\mathbf{I}$, and that $\mathbf{I}$ is covariantly constant. Thus the manifold is Kähler. In the same way, if we require (5.69) to be invariant under the three extra supersymmetries (5.63), we find that the manifold is hyperkähler. Indeed, one can use these arguments to prove the following classification theorem: For irreducible target manifolds, the action (5.69) has $N=1$ supersymmetry for an arbitrary Riemannian manifold, has $N=2$ supersymmetry if and only if the manifold is Kähler [28], and has $N=4$ supersymmetry if and only if the manifold is hyperkähler [29]. The supersymmetries beyond $N=1$ are associated with complex 
Table 2. Theorem due to Zumino [28] and Alvarez-Gaumé and Freedman [29] relating the number of supersymmetries on the base space and the geometry of the target space

\begin{tabular}{ll}
\hline Number of supersymmetries & Type of manifold \\
\hline 1 & Riemannian \\
2 & Kähler \\
3 or 4 & Hyperkähler \\
\hline
\end{tabular}

structures on the manifold. Thus $N=3$ supersymmetry implies $N=4$ because two anticommuting complex structures imply the existence of a third. We summarize the theorem in Table $2 .{ }^{5}$

$N=2$ supersymmetric actions for chiral superfields have the form [28]

$$
S=\int d^{3} x D^{2} \bar{D}^{2} K(\Phi, \bar{\Phi}),
$$

where $K$ is a real function of $\Phi$ and $\bar{\Phi}$. This describes a nonlinear $\sigma$-model if and only if $K$ depends algebraically on $\Phi$ and $\bar{\Phi}$ but not on their derivatives. Computing the component or $N=1$ action one discovers that this has the form (5.70) or (5.69) where the metric is a Kähler metric with Kähler potential $K$.

One can also add nonderivative interaction terms to (5.72). These involve an arbitrary holomorphic function $P(\Phi)$ and have the form

$$
S_{\text {INT }}=\int d^{3} x\left(D^{2} P(\Phi)+\bar{D}^{2} \bar{P}(\bar{\Phi})\right) .
$$

Note that the nonderivative interaction terms involve a chiral superspace "measure" $D^{2}$ rather than $D^{2} \bar{D}^{2}$ as in (5.72).

If we impose invariance of the action (5.72) under the two additional supersymmetry transformations (5.64) we recover the condition that $K$ is the Kähler potential of a hyperkähler manifold. We then also find an expression for $X^{P}$ in (5.64) [3]:

$$
X^{P}=-\frac{1}{2} K_{r}(\mathbf{J}-i \mathbf{K})_{\bar{q}}^{r} g^{\bar{q} p} .
$$

This gives the $N=4$ supersymmetry in terms of the complex structures in $N=2$ superspace.

Superspace field equations can be derived from superspace actions by formally (because the action involves anticommuting quantities) extremizing with respect to the superfields. Care must be taken to first express constrained superfields $(5.36,39)$ in terms of unconstrained ones $(5.37,41)$.

\section{The Supersymmetric Construction of Hyperkähler Metrics}

\section{(A) The Supersymmetric Legendre Transform Construction}

The Legendre transform construction of hyperkähler manifolds of Sect. 2 originates in the correspondence between $N=4$ supersymmetric nonlinear $\sigma$-models and hyperkähler manifolds discussed in Sect. 5. Here we give this

\footnotetext{
${ }^{5}$ The classification depends on the dimension of the spacetime $M$, see, e.g., [8]
} 
supersymmetric construction [5]. It is a generalization of the duality of Sect. 4B to a supersymmetric Legendre transform that interchanges chiral and linear $N=2$ superfields.

Duality in $N=1$ superspace closely parallels the bosonic duality. The general supersymmetric $\sigma$-model action is (5.69)

$$
S=-\frac{1}{2} \int d^{3} x D^{2}\left(g_{i j}(\Phi) D^{\alpha} \Phi^{i} D_{\alpha} \Phi^{j}\right) .
$$

The component action (5.70) shows that $g_{i j}\left(\Phi^{k} \mid\right)$ is a metric of a bosonic nonlinear $\sigma$-model. Hence any bosonic $\sigma$-model in (three dimensions) has an $N=1$ supersymmetric extension. We assume the existence of a non-null Killing vector field and choose coordinates $\Phi$ such that $g_{i j}$ is independent of $\Phi^{0}$. A first order action is [cf. (4.8)]

$$
S=-\frac{1}{2} \int d^{3} x D^{2}\left(g_{00} \Gamma^{\alpha} \Gamma_{\alpha}+2 g_{0 i} \Gamma^{\alpha} D_{\alpha} \Phi^{i}+g_{i j} D^{\alpha} \Phi^{i} D_{\alpha} \Phi^{j}+T^{\alpha} D^{\beta} D_{\alpha} \Gamma_{\beta}\right),
$$

where $i, j \neq 0$ and $\Gamma^{\alpha}$ and $T^{\alpha}$ are spinor superfields. (Formally) extremizing (6.2) w.r.t. $T^{\alpha}$ gives

$$
D^{\beta} D_{\alpha} \Gamma_{\beta}=0,
$$

which is solved by [see (5.18)]

$$
\Gamma_{\alpha}=D_{\alpha} \Phi^{0}
$$

where $\Phi^{0}$ is a general real scalar superfield. Substituting (6.4) in (6.2) we recover (6.1). Extremizing w.r.t. $\Gamma_{\alpha}$ [cf. (4.9)] and substituting the result in (6.2) we find the dual action [cf. (4.10)]

$$
\begin{aligned}
S= & -\frac{1}{2} \int d^{3} x D^{2}\left[\left(g_{00}\right)^{-1}\left(\frac{1}{4} F^{\alpha} F_{\alpha}+i F^{\alpha} g_{0 i} D_{\alpha} \Phi^{i}\right)\right. \\
& \left.+\left(g_{i j}-\left(g_{00}\right)^{-1} g_{0 i} g_{0 j}\right) D^{\alpha} \Phi^{i} D_{\alpha} \Phi^{i}\right]
\end{aligned}
$$

with $F_{\alpha}=i D^{\beta} D_{\alpha} T_{\beta}(5.22)$. The actions (6.5) and (6.1) are related via a Legendre transform w.r.t. $D^{\alpha} \Phi^{0}$.

A supersymmetric nonlinear $\sigma$-model has $N=2$ supersymmetry if and only if the target space $F$ is Kähler (see Table 2). The action in terms of $N=2$ superfields is then (5.72)

$$
S=\int d^{3} x D^{2} \bar{D}^{2} K\left(\Phi^{p}, \bar{\Phi}^{\bar{q}}\right),
$$

where $K$ is the Kähler potential. Expanding (6.6) in components using $(5.36,43)$ we recognize a supersymmetric extension of the bosonic $\sigma$-model with metric $\left(\partial^{2} K / \partial \Phi^{p} \partial \bar{\Phi}^{\bar{q}}\right) \mid$. We assume the existence of a $U(1)$ isometry generated by a holomorphic Killing vector (1.4-8) and choose coordinates such that $K=K\left(\Phi^{0}+\bar{\Phi}^{0}, \Phi^{p}, \bar{\Phi}^{\bar{q}}\right), p, q \neq 0$. A first order action is

$$
S=\int d^{3} x D^{2} \bar{D}^{2}\left[K\left(V, \Phi^{p}, \bar{\Phi}^{\bar{q}}\right)-V G\right],
$$

where $V$ is a real and $G$ a linear superfield (5.39). Extremizing (6.7) w.r.t. $G$ gives [using (5.41)]

$$
\bar{D}^{\alpha} D_{\alpha} V=0,
$$

which implies

$$
V=\Phi^{0}+\bar{\Phi}^{\overline{0}}
$$


Substituting (6.9) in (6.7) we recover (6.6). Extremizing w.r.t. $V$ we find

$$
\frac{\partial K}{\partial V} \equiv K_{V}=-G
$$

This determines $V$ as a function of $G$ (and $\Phi^{p}, \bar{\Phi}^{\bar{q}}, p, q \neq 0$ ). The action obtained by substituting $V\left(G, \Phi^{p}, \bar{\Phi}^{\bar{q}}\right)$ into (6.7) is

$$
S=\int d^{3} x D^{2} \bar{D}^{2}\left[K\left(V, \Phi^{p}, \bar{\Phi}^{\bar{q}}\right)-V G\right]=\int d^{3} x D^{2} \bar{D}^{2} f\left(G, \Phi^{p}, \bar{\Phi}^{\bar{q}}\right) .
$$

The relation of $K$ to $f$ is via a Legendre transform. The Legendre transform from $f$ to $K$ can be performed for any $f\left(G, \Phi^{p}, \bar{\Phi}^{\bar{q}}\right)$ while the transform from $K$ to $f$ requires the existence of a holomorphic Killing vector. For a general isometry group acting on $M$ the Legendre transform can be carried out for each commuting holomorphic Killing vector [22].

A supersymmetric nonlinear $\sigma$-model has $N=4$ supersymmetry if and only if the target space $F$ is hyperkähler (see Table 2). The action in terms of $N=2$ superfields is the action (6.6) with the additional requirement that $K$ is a Kähler potential for a hyperkähler manifold. The two nonmanifest supersymmetry transformations are given by (5.64). In this case we find it more instructive to describe the dualization starting from the $N=4$ linear multiplet, which consists of an $N=2$ chiral superfield $\chi$ and an $N=2$ linear superfield $G$. The action for a set of linear multiplets is

$$
S_{G}=\int d^{3} x D^{2} \bar{D}^{2} f\left(G^{i}, \chi^{i}, \bar{\chi}^{i}\right) .
$$

It is invariant under the two nonmanifest supersymmetries

$$
\delta \chi^{i}=\bar{D}^{2}\left(\bar{\eta} G^{i}\right), \quad \delta G^{i}=-D^{\alpha}\left(\chi^{i} D_{\alpha} \eta\right)-\bar{D}^{\alpha}\left(\bar{\chi}^{i} \bar{D}_{\alpha} \bar{\eta}\right)
$$

[where $\eta$ obeys (5.65)] if and only if

$$
f_{G^{i} G^{j}}+f_{\bar{\chi}^{i} \chi^{j}}=0, \quad \forall i, j
$$

This is a set of linear partial differential equations; for one $N=4$ multiplet (6.14) reduces to the three dimensional Laplace equation.

A first order action is

$$
S=\int d^{3} x D^{2} \bar{D}^{2}\left[f\left(\Psi^{i}, \chi^{i}, \bar{\chi}^{i}\right)-\Psi^{i}\left(\Phi^{i}+\bar{\Phi}^{i}\right)\right] .
$$

Extremizing $S$ w.r.t. $\Phi$ and $\bar{\Phi}$ we find that $\Psi^{i}$ are linear $\left(\Psi^{i}=G^{i}\right)$ and recover (6.12). Extremizing w.r.t. $\Psi$ we find

$$
f_{\Psi^{i}}=\Phi^{i}+\bar{\Phi}^{i}
$$

Solving for $\Psi^{i}, \Psi^{i}=\Psi^{i}\left(\Phi^{j}+\bar{\Phi}^{j}, \chi^{k}, \bar{\chi}^{k}\right)$, and substituting into $S$ gives

$$
S_{\Phi}=\int d^{3} x D^{2} \bar{D}^{2}\left[f\left(\Psi^{i}, \chi^{j}, \bar{\chi}^{j}\right)-\left(\Phi^{i}+\bar{\Phi}^{i}\right) \Psi^{i}\right] \equiv \int d^{3} x D^{2} \bar{D}^{2} K\left(\Phi^{j}+\bar{\Phi}^{j}, \chi^{i}, \bar{\chi}^{i}\right) .
$$

This is an $N=4$ supersymmetric nonlinear $\sigma$-model with $n$ abelian holomorphic isometries. $K$ is the Legendre transform of $f$. The nonmanifest supersymmetries are

$$
\begin{gathered}
\delta \chi^{i}=-\bar{D}^{2}\left(\bar{\eta} K_{\Phi^{i}}\right), \\
\delta \Phi^{i}=\bar{D}^{2}\left\{\bar{\eta}\left[K_{\chi^{i}}+K_{\Phi^{j}}\left(K_{\chi^{j} \bar{\Phi}^{k}}\left(K_{\Phi^{k} \bar{\Phi}^{i}}\right)^{-1}-K_{\chi^{i} \bar{\Phi}^{k}}\left(K_{\bar{\Phi}^{k} \Phi^{j}}\right)^{-1}\right)\right]\right\}
\end{gathered}
$$


and the constraint (6.14) becomes

$$
K_{\chi^{i} \bar{\chi}^{j}}=\left(K_{\Phi^{i} \bar{\Phi}^{j}}\right)^{-1}+K_{\chi^{i} \bar{\Phi}^{m}}\left(K_{\Phi^{m} \bar{\Phi}^{n}}\right)^{-1} K_{\Phi^{n} \bar{\chi}^{j}} .
$$

This is a set of nonlinear partial differential equations. For one $N=4$ linear multiplet (6.19) is the Monge-Amperé equation.

According to Table 1 in Sect. 5 the action (6.17) gives $4 n$ (real) dimensional hyperkähler manifolds. These are obtained by solving the linear partial differential equations (6.14). Note that the constraint (6.19) on $K$ corresponding to (6.14) is nonlinear. The two noncanonical (integrable) complex structures $\mathbf{J}$ and $\mathbf{K}$ can be read off from the supersymmetry transformations (6.18) by comparison to $(5.64,66)$; this is how $(2.8)$ was found.

The hyperkähler manifold has $n$ abelian holomorphic isometries. It can be shown that these are triholomorphic (1.14) [22], and that all $4 n$ (real) dimensional hyperkähler manifolds with $n$ abelian triholomorphic isometries can be obtained via the Legendre transform.

\section{(B) Gauging of Isometries and the Quotient Construction}

In this section we discuss $N=1,2$, and 4 supersymmetric extensions of the gauging procedure and quotient construction of Sect. $4 \mathrm{C}$. The $N=1$ quotient leads to an ordinary quotient (Sect. 3). The $N=2$ quotient automatically leads to the Kähler quotient of Sect. 2B [3]. This follows directly because we work in $N=2$ superspace, and thus supersymmetry is preserved at every stage of the construction and the classification theorem in Sect. 5 implies that the quotient manifold is Kähler. A key feature is the complex action of the gauge group on chiral superfields. The $N=4$ construction is a simple extension from $N=2$; however, since we do not use $N=4$ superfields, the $N=4$ supersymmetry has to be verified explicitly [3].

The gauging of isometries of $N=1 \sigma$-models precisely parallels the gauging described in Sect. 4C. We consider a supersymmetric $\sigma$-model with action (5.69)

$$
S=-\frac{1}{2} \int d^{3} x D^{2}\left[g_{i j}(\Phi) D^{\alpha} \Phi^{i} D_{\alpha} \Phi^{j}\right],
$$

where the metric $g_{i j}$ is invariant under isometries generated by a vector field $X$ [cf. $(1.5-7)]$

$$
X \Phi^{i}=X^{A} k_{A}^{i} .
$$

Note that $k_{A}^{i}\left(\Phi^{j}\right)$ are functions of superfields. Using minimal coupling, (4.14), we obtain the gauged action by replacing

$$
D_{\alpha} \Phi^{i} \rightarrow \nabla_{\alpha} \Phi^{i} \equiv D_{\alpha} \Phi^{i}+\Gamma_{\alpha}^{A} k_{A}^{i},
$$

where $\Gamma_{\alpha}^{A}$ is the spinor component superfield of a connection super one-form [see (5.25)]. We take the quotient by formally extremizing the action w.r.t. $\Gamma_{\alpha}^{A}$. We find [cf. (4.16)]

$$
\Gamma_{\alpha}^{A}=-g_{i j} k_{B}^{j} H^{A B} D_{\alpha} \Phi^{i}, \quad H^{A B}=\left(g_{i_{j}} k_{A}^{i} k_{B}^{j}\right)^{-1} ;
$$

$\Gamma_{\alpha}^{A}$ is the pullback to superspace of the connection discussed in Sect. 3A. Substituting (6.23) into the gauged action, we find the nonlinear $\sigma$-model with the induced metric for the quotient manifold

$$
S=-\frac{1}{2} \int d^{3} x D^{2}\left[\left(g_{i j}-g_{i m} g_{j n} k_{A}^{m} k_{B}^{n} H^{A B}\right) D^{\alpha} \Phi^{i} D_{\alpha} \Phi^{j}\right] .
$$


This construction gives the ordinary quotient on the bosonic components (4.17), and it constrains the fermions to lie in the tangent space of this quotient manifold.

The action of an $N=2$ supersymmetric $\sigma$-model is (5.72)

$$
S=\int d^{3} x D^{2} \bar{D}^{2} K(\Phi, \bar{\Phi}),
$$

where $\Phi$ and $\bar{\Phi}$ are chiral superfields corresponding to holomorphic and antiholomorphic coordinates $z$ and $\bar{z}$. When we attempt to gauge the holomorphic isometries $(1.4,5)$, since there are no explicit derivatives in (6.25), we can not use the minimal coupling prescription (6.22).

Under an isometry the Kähler potential $K$ transforms as

$$
X K=v^{X}+\bar{v}^{X},
$$

where $v^{X}=X^{A} v_{A}(\Phi)$ is a holomorphic function. From (1.10) it follows that

$$
\frac{1}{2}(X-i \mathbf{I} X) K \equiv X^{A} k_{A}^{p} K_{, p}=v^{X}-i \mu^{X},
$$

where $\mu^{X}$ is the Hamiltonian function. As discussed in (5.54-56), in $N=2$ supersymmetric systems, the gauge group $G$ acts through its complexification $G^{C}$ as a consequence of the chiral structure of $N=2$ superspace. The basic gauge field in $N=2$ superspace is a hermitian Lie-algebra valued superfield potential $V=V^{A} T_{A}$, where $T_{A}$ are the Lie-algebra generators. We define the superfield generalization of (2.21),

$$
Y=-\frac{1}{2} \mathbf{I} V=-\frac{i}{2} V^{A}\left(k_{A}^{p} \partial_{p}-\bar{k}_{A}^{\bar{p}} \bar{\partial}_{\bar{p}}\right) .
$$

To gauge the action [3], we introduce an extra complex coordinate $\zeta(=(1 / 4 \pi) \ln s$ in the language of Sect. 3E), and let it transform as [cf. (3.43)]

$$
\delta_{X} \zeta=v^{X} .
$$

That this is an action (i.e., a realization of the symmetry group) follows immediately from (6.27) and the equivariance of $\mu$ [cf. $(1.7,10,11)]$ :

$$
\begin{aligned}
{\left[\delta_{X}, \delta_{Y}\right] \zeta } & =X v^{Y}-Y v^{X}=\frac{1}{2}(X-i \mathbf{I} X) v^{Y}-X \leftrightarrow Y \\
& =\left(X^{A} Y^{B}-X^{B} Y^{A}\right) k_{A}^{q}\left(\left(k_{B}^{p} K_{p}\right)_{q}+i \mu_{B, q}\right) \\
& =X^{A} Y^{B}\left(k_{[A}^{q} k_{B], q}^{p} K_{p}+i k_{[A}^{q} \mu_{B], q}\right) \\
& =X^{A} Y^{B} c_{A B}{ }^{D}\left(k_{D}^{p} K_{p}+i \mu_{D}\right) \\
& =v^{[X, Y]} .
\end{aligned}
$$

Clearly, this gives a realization of the entire complexified group $(\zeta$ is a holomorphic coordinate). We now define

$$
\tilde{K}=K-\zeta-\bar{\zeta}
$$

$\left(\tilde{K}=\frac{1}{4 \pi} \ln h\right.$ of Sect. $\left.3 \mathrm{E}\right)$. This is manifestly invariant under the real group [but transforms under the complex group as $\mathbf{I} X \tilde{K}=2 \mu^{X}$, cf. (3.51)]. As $\tilde{K}$ is invariant, it is sufficient to go to a representation where all fields transform with the same 
parameter. This we do with $Y$, and write the gauged $\widetilde{K}$ as

$$
\hat{\tilde{K}}=e^{Y} \tilde{K}=K(\Phi, \bar{\Phi})-\int_{0}^{1} d t e^{t Y} \mu^{V}-\zeta-\bar{\zeta}
$$

Since the coordinate $\zeta$ is holomorphic, it can be dropped. Then the gauged action is given by the super Lagrange density [cf. (2.23)]

$$
\widehat{K}(\Phi, \bar{\Phi}, V)=K(\Phi, \bar{\Phi})-\int_{0}^{1} d t e^{t Y} \mu^{V},
$$

where $\mu^{V}=V^{A} \mu_{A}$. Again we take the quotient by formally extremizing w.r.t. the gauge potential, in this case $V^{A}$. We find

$$
e^{Y} \mu_{A}=0
$$

which is to be solved for $V(\Phi, \bar{\Phi})$. The Kähler potential $\hat{K}(\Phi, \bar{\Phi}, V(\Phi, \bar{\Phi}))$ is defined [up to irrelevant holomorphic and antiholomorphic pieces, cf. (6.26)] on the orbit space $\hat{M}=M / G^{C}$ of the complexified group. This is the symplectic (Kähler) quotient discussed in Sect. 3.

As an example of the construction we consider $C P(1)$. We start from the flat space Kähler potential for $C^{2}$

$$
K(\Phi, \bar{\Phi})=\Phi^{p} \bar{\Phi}^{\bar{p}}, \quad p=1,2 .
$$

We consider the $U(1)$ isometry generated by

$$
X \Phi^{p}=i \xi \Phi^{p}, \quad X \bar{\Phi}^{\bar{p}}=-i \xi \bar{\Phi}^{\bar{p}} .
$$

(Since this isometry has a fixed point, $v^{\xi}+\bar{v}^{\xi}$ vanishes.) The moment map has the special form $\mu^{\xi}=-\xi(K-c)$, where $c$ is the arbitrary real constant discussed in Sect. 1. We find the gauged Lagrange density (6.33)

$$
\hat{K}(\Phi, \bar{\Phi}, V)=\Phi^{p} \bar{\Phi}^{\bar{p}} e^{V}-c V .
$$

Extremizing w.r.t. $V$ gives

$$
e^{V} \Phi^{p} \bar{\Phi}^{\bar{p}}=c \Rightarrow V=-\ln \left(\frac{\Phi^{p} \bar{\Phi}^{\bar{p}}}{c}\right)
$$

and hence the Kähler potential on the quotient manifold is

$$
\widehat{K}(\Phi, \bar{\Phi}, V(\Phi, \bar{\Phi}))=c\left[\ln \left(\frac{\Phi^{p} \bar{\Phi}^{\bar{p}}}{c}\right)+1\right]=c \ln (1+\zeta \bar{\zeta})+f(\Phi)+\bar{f}(\bar{\Phi}),
$$

where we have introduced inhomogenous coordinates $\zeta=\Phi^{1} / \Phi^{2}$ and $f=c\left(\ln \left(\Phi^{2} / \sqrt{2}\right)+1 / 2\right)$ is an irrelevant holomorphic function. The Kähler potential $\hat{K}$ in (6.39) is the familiar one for $C P(1)$.

We now turn to the $N=4$ construction. As discussed in Sect. 5, an $N=4$ supersymmetric $\sigma$-model can be described in terms of $N=2$ superfields and has the action (6.25), where now $K$ is the Kähler potential of a hyperkähler manifold. The description in terms of $N=2$ superfields selects one of the complex structures to have its canonical form (1.1). Corresponding to this canonical complex structure there is a real moment map $\mu^{(1)}$ for each holomorphic isometry (1.10). From the 
remaining complex structures we also construct a holomorphic and an antiholomorphic moment map $\mu^{+}$and $\mu^{-}$for each triholomorphic isometry (1.15). As discussed in Sect. 5, the $N=4$ gauge multiplet is described by an $N=2$ gauge multiplet $V$ and an $N=2$ chiral multiplet $S$. The gauged action is

$$
S=\int d^{3} x\left[D^{2} \bar{D}^{2} \hat{K}(\Phi, \bar{\Phi}, V)+\frac{1}{2} D^{2} \mu_{+}^{S}+\frac{1}{2} \bar{D}^{2} \mu_{-}^{\bar{S}}\right],
$$

where $\hat{K}$ is given by (6.33) and $\mu_{+}^{S}=S^{A} \mu_{A}^{+}$. The crucial point is that the action (6.40) is $N=4$ supersymmetric: The nonmanifest supersymmetries are given by (5.67) and the gauge covariantizations of (5.64)

$$
\delta \Phi^{p}=\bar{D}^{2}\left(\bar{\eta} e^{Z} X^{p}\right), \quad \delta \bar{\Phi}^{\bar{p}}=D^{2}\left(\eta e^{\bar{Z}} \bar{X}^{\bar{p}}\right), \quad Z \equiv i V^{A} k_{A}^{p} \partial_{p} .
$$

Extremizing (6.40) w.r.t. $V$ gives the condition (6.35) as before; extremizing w.r.t. $S$ and $\bar{S}$ gives

$$
\mu_{+}=\mu_{-}=0 .
$$

This is the hyperkähler quotient of Sect. 2. By the classification theorem (Table 2) the hyperkähler property is ensured by the $N=4$ supersymmetry of the action. The noncanonical complex structures $\mathbf{J}$ and $\mathbf{K}$ can be read off from the supersymmetry transformations $(6.41)$ [cf. $(5.64,66)]$.

Acknowledgements. N.J.H., A.K., and U.L. thank the ITP and the Mathematics Department at Stony Brook and M.R. the ITP at Stockholm for hospitality over the years during which this paper was written.

\section{References}

1. Alvarez-Gaumé, L., Freedman, D.Z.: In: Unification of the fundamental particle interactions. Ferrara, S., Ellis, J., van Nieuwenhuizen, P. (eds.). New York: Plenum 1980

Yano, K.: Differential geometry on complex and almost complex spaces. Oxford: Pergamon 1965

Yano, K., Kon, M.: Structures on manifolds. World Scientific 1984

2. Kobayashi, S., Nomizu, K.: Foundations of differential geometry, Vol. II. New York: Wiley 1969

3. Hull, C.M., Karlhede, A., Lindström, U., Roček, M.: Nonlinear $\sigma$-models and their gauging in and out of superspace. Nucl. Phys. B 266, 1 (1986)

4. Curtright, T.L., Freedman, D.Z.: Nonlinear $\sigma$-models with extended supersymmetry in four dimensions. Phys. Lett. 90 B, 71 (1980)

Roček, M., Townsend, P.K.: Three-loop finiteness of the $N=4$ supersymmetric non-linear $\sigma$-model. Phys. Lett. 96B, 72 (1980)

5. Lindström, U., Roček, M.: Scalar tensor duality and $N=1,2$ nonlinear $\sigma$-models. Nucl. Phys. B 222, 285 (1983)

6. Bagger, J., Witten, E.: Quantization of Newton's constant in certain supergravity theories. Phys. Lett. B 115, 202 (1982)

7. Karlhede, A., Lindström, U., Roček, M.: Self-interacting tensor multiplets in $N=2$ superspace. Phys. Lett. 147 B, 297 (1984)

8. Roček, M.: Supersymmetry and nonlinear $\sigma$-models. Physica 15D, 75 (1985)

9. Eguchi, T., Hanson, A.J.: Self-dual solutions to Euclidean gravity. Ann. Phys. 120, 82 (1979)

10. Gibbons, G.W., Hawking, S.W.: Classification of gravitational instanton symmetries. Commun. Math. Phys. 66, 291 (1979)

11. Calabi, E.: Métriques Kählériennes et fibrés holomorphes. Ann. Sci. Ec. Norm. Super. 12, 269 (1979) 
12. Guillemin, V., Sternberg, S.: Symplectic techniques in physics. Cambridge: Cambridge University Press 1984

13. Kirwan, F.: Cohomology of quotients in algebraic and symplectic geometry. Princeton Mathematical Notes, Vol. 31. Princeton, NJ: Princeton University Press 1985

14. Penrose, R.: Nonlinear gravitons and curved twistor theory. Gen. Relativ. Gravitation 7, 31 (1976)

15. Salamon, S.: Quaternionic Kähler manifolds. Invent. Math. 67, 143 (1982)

16. Salamon, S.: Invent. differential geometry of quaternionic manifold. Ann. Sci. Ec. Norm. Super. (to appear)

17. Newlander, A., Nirenberg, L.: Complex analytic coordinates in almost complex manifolds. Ann. Math. 65, 391 (1958)

18. Kodaira, K.: A theorem of completeness of characteristic systems for analytic families of compact submanifolds of complex manifolds. Ann. Math. 84, 146 (1962)

19. Meetz, K.: Realization of chiral symmetry in a curved isospin space. J. Math. Phys. 10, 589 (1969)

Honerkamp, J.: Chiral multi-loops. Nucl. Phys. B 36, 130 (1972)

Friedan, D.: Nonlinear models in $2+\varepsilon$ dimensions. Phys. Rev. Lett. 45, 1057 (1980); Nonlinear models in $2+\varepsilon$ dimensions. Ann. Phys. 163, 318 (1985)

20. Freedman, D.Z., van Nieuwenhuizen, P.: Properties of supergravity theory. Phys. Rev. D 14, 912 (1976)

21. Fradkin, E.S., Tseytlin, A.A.: Quantum equivalence of dual field theories. Ann. Phys. 162, 31 (1985)

22. Howe, P.S., Karlhede, A., Lindström, U., Roček, M.: The geometry of duality. Phys. Lett. 168B, 89 (1986)

23. Ogievietsky, V., Mezincescu, L.: Boson fermion symmetries and superfields. Usp. Fiz. Nauk. 117, 636 (1975) (Sov. Phys. Usp. 18, 960 (1976))

Fayet, P., Ferrara, S.: Supersymmetry. Phys. Rep. 32C, 250 (1977)

Salam, A., Strathdee, J.: Supersymmetry and superfields. Fortschr. Phys. 26, 5 (1978)

van Nieuwenhuizen, P.: Supergravity. Phys. Rep. 68(4), 189 (1981)

Wess, J., Bagger, J.: Supersymmetry and supergravity. Princeton, NJ: Princeton University Press 1983

Sohnius, M.: Introducing supersymmetry. Phys. Rep. 128(2, 3), 41 (1985)

24. Gates, S.J., Grisaru, M.T., Roček, M., Siegel, W.: Superspace, or one thousand and one lectures in supersymmetry. Reading, MA: Benjamin/Cummings 1983

25. Gates, S.J., Hull, C.M., Roček, M.: Twisted multiplets and new supersymmetric non-linear $\sigma$-models. Nucl. Phys. B 248, 157 (1984)

26. Galperin, A., Ivanov, E., Kalitzin, S., Ogievietsky, V., Sokatchev, E.: Unconstrained $N=2$ matter Yang-Mills and supergravity theories in harmonic superspace. Class. Quant. Grav. 1, 496 (1984)

Galperin, A., Ivanov, E., Ogievietsky, V., Sokatchev, E.: Hyperkähler metrics and harmonic superspace. Dubna preprint 1985

27. Siegel, W.: Unextended superfields in extended supersymmetry. Nucl. Phys. B 156, 135 (1979)

28. Zumino, B.: Supersymmetry and Kähler manifolds. Phys. Lett. 87 B, 203 (1979)

29. Alvarez-Gaumé, L., Freedman, D.Z.: Geometrical structure and ultraviolet finiteness in the supersymmetric $\sigma$-model. Commun. Math. Phys. 80, 443 (1981)

Communicated by L. Alvarez-Gaumé

Received August 8, 1986 
